$10-2-2017$

\title{
Overview of NSTX Upgrade initial results and modelling highlights
}

\author{
J. E. Menard \\ jmenard@pppl.gov \\ J. P. Allain \\ D. J. Battaglia \\ F. Bedoya \\ R. E. Bell
}

See next page for additional authors

Follow this and additional works at: https://scholarworks.wm.edu/aspubs

\section{Recommended Citation}

Menard, J. E.; Allain, J. P.; Battaglia, D. J.; Bedoya, F.; Bell, R. E.; Belova, E.; Berkery, J. W.; Boyer, M. D.; Crocker, N.; Diallo, A.; Ebrahimi, F.; Ferraro, N.; Fredrickson, E.; Frerichs, H.; Gerhardt, S.; Gorelenkov, N.; Guttenfelder, W.; Heidbrink, W.; Kaita, R.; Kaye, S. M.; Kriete, D. M.; Kubota, S.; LeBlanc, B. P.; Liu, D.; and Meier, E., Overview of NSTX Upgrade initial results and modelling highlights (2017). NUCLEAR FUSION, 57(10).

10.1088/1741-4326/aa600a

This Article is brought to you for free and open access by the Arts and Sciences at W\&M ScholarWorks. It has been accepted for inclusion in Arts \& Sciences Articles by an authorized administrator of W\&M ScholarWorks. For more information, please contact scholarworks@wm.edu. 


\section{Authors}

J. E. Menard, J. P. Allain, D. J. Battaglia, F. Bedoya, R. E. Bell, E. Belova, J. W. Berkery, M. D. Boyer, N. Crocker, A. Diallo, F. Ebrahimi, N. Ferraro, E. Fredrickson, H. Frerichs, S. Gerhardt, N. Gorelenkov, W. Guttenfelder, W. Heidbrink, R. Kaita, S. M. Kaye, D. M. Kriete, S. Kubota, B. P. LeBlanc, D. Liu, and E. Meier 


\section{UC Irvine}

\section{UC Irvine Previously Published Works}

Title

Overview of NSTX Upgrade initial results and modelling highlights

Permalink

https://escholarship.org/uc/item/5f1419gd

Journal

Nuclear Fusion, 57(10)

\section{ISSN}

0029-5515

\section{Authors}

Menard, JE

Allain, JP

Battaglia, DJ

et al.

\section{Publication Date}

2017-06-20

DOI

10.1088/1741-4326/aa600a

License

CC BY 4.0

Peer reviewed 


\title{
Overview of NSTX Upgrade initial results and modelling highlights
}

\author{
J.E. Menard ${ }^{1}$, J.P. Allain ${ }^{2}$, D.J. Battaglia ${ }^{1}$, F. Bedoya ${ }^{2}$, R.E. Bell ${ }^{1}$, E. Belova ${ }^{1}$, \\ J.W. Berkery ${ }^{3}$, M.D. Boyer ${ }^{1}$, N. Crocker $^{4}$, A. Diallo ${ }^{1}$, F. Ebrahimi ${ }^{1}$, \\ N. Ferraro ${ }^{1}$, E. Fredrickson ${ }^{1}$, H. Frerichs ${ }^{5}$, S. Gerhardt ${ }^{1}$, N. Gorelenkov ${ }^{1}$, \\ W. Guttenfelder ${ }^{1}$, W. Heidbrink ${ }^{6}$, R. Kaita ${ }^{1}$, S.M. Kaye ${ }^{1}$, D.M. Kriete ${ }^{5}$, \\ S. Kubota ${ }^{4}$, B.P. LeBlanc ${ }^{1}$, D. Liu ${ }^{6}$, R. Lunsford ${ }^{1}$, D. Mueller ${ }^{1}$, C.E. Myers ${ }^{1}$, \\ M. Ono ${ }^{1}$, J.-K. Park ${ }^{1}$, M. Podesta ${ }^{1}$, R. Raman ${ }^{7}$, M. Reinke ${ }^{8}$, Y. Ren ${ }^{1}$, \\ S.A. Sabbagh ${ }^{3}$, O. Schmitz ${ }^{5}$, F. Scotti ${ }^{9}$, Y. Sechrest ${ }^{10}$, C.H. Skinner ${ }^{1}$, \\ D.R. Smith ${ }^{5}$, V. Soukhanovskii ${ }^{9}$, T. Stoltzfus-Dueck ${ }^{1}$, H. Yuh ${ }^{10}$, Z. Wang ${ }^{1}$, \\ I. Waters ${ }^{5}$, J.-W. Ahn ${ }^{8}$, R. Andre ${ }^{1}$, R. Barchfeld ${ }^{11}$, P. Beiersdorfer ${ }^{9}$, \\ N. Bertelli ${ }^{1}$, A. Bhattacharjee ${ }^{1}$, D. Brennan ${ }^{12}$, R. Buttery ${ }^{13}$, A. Capece $^{14}$, \\ G. Canal ${ }^{13}$, J. Canik ${ }^{8}$, C.S. Chang ${ }^{1}$, D. Darrow ${ }^{1}$, L. Delgado-Aparicio ${ }^{1}$, \\ C. Domier ${ }^{11}$, S. Ethier ${ }^{1}$, T. Evans ${ }^{13}$, J. Ferron ${ }^{13}$, M. Finkenthal ${ }^{15}$, R. Fonck ${ }^{5}$, \\ K. Gan ${ }^{16}$, D. Gates ${ }^{1}$, I. Goumiri ${ }^{5}$, T. Gray ${ }^{8}$, J. Hosea ${ }^{1}$, D. Humphreys ${ }^{13}$, \\ T. Jarboe ${ }^{7}$, S. Jardin ${ }^{1}$, M.A. Jaworski ${ }^{1}$, B. Koel ${ }^{12}$, E. Kolemen ${ }^{12}$, S. Ku ${ }^{1}$, \\ R.J. La Haye ${ }^{13}$, F. Levinton ${ }^{10}$, N. Luhmann ${ }^{11}$, R. Maingi ${ }^{1}$, R. Maqueda ${ }^{17}$, \\ G. McKee ${ }^{5}$, E. Meier ${ }^{18}$, J. Myra ${ }^{19}$, R. Perkins ${ }^{1}$, F. Poli ${ }^{1}$, T. Rhodes ${ }^{4}$, \\ J. Riquezes ${ }^{20}$, C. Rowley ${ }^{12}$, D. Russell ${ }^{19}$, E. Schuster ${ }^{21}$, B. Stratton ${ }^{1}$, \\ D. Stutman ${ }^{15}$, G. Taylor ${ }^{1}$, K. Tritz ${ }^{15}$, W. Wang ${ }^{1}$, B. Wirth ${ }^{16}$ and S.J. Zweben ${ }^{1}$
}

1 Princeton Plasma Physics Laboratory, Princeton University, Princeton, NJ 08543, United States of America

${ }^{2}$ University of Illinois at Urbana-Champaign, Urbana, IL, United States of America

${ }^{3}$ Columbia University, New York, NY, United States of America

${ }^{4}$ University of California at Los Angeles, Los Angeles, CA, United States of America

${ }^{5}$ University of Wisconsin, Madison, WI, United States of America

${ }^{6}$ University of California at Irvine, Irvine, CA, United States of America

7 University of Washington, Seattle, WA, United States of America

8 Oak Ridge National Laboratory, Oak Ridge, TN, United States of America

9 Lawrence Livermore National Laboratory, Livermore, CA, United States of America

${ }_{10}$ Nova Photonics, Princeton, NJ, United States of America

11 University of California at Davis, Davis, CA, United States of America

12 Princeton University, Princeton, NJ, United States of America

13 General Atomics, San Diego, CA, United States of America

14 The College of New Jersey, Ewing, NJ, United States of America

15 Johns Hopkins University, Baltimore, MD, United States of America

16 University of Tennessee, Knoxville, TN, United States of America

17 X Science LLC, Plainsboro, NJ, United States of America

18 The College of William and Mary, Williamsburg, VA, United States of America

19 Lodestar Research Corporation, Boulder, CO, United States of America

${ }^{20}$ University of Michigan, Ann Arbor, MI, United States of America

${ }^{21}$ Lehigh University, Bethlehem, PA, United States of America

E-mail: jmenard@pppl.gov 


\section{Abstract \\ The National Spherical Torus Experiment (NSTX) has undergone a major upgrade, and the NSTX Upgrade (NSTX-U) Project was completed in the summer of 2015. NSTX-U first plasma was subsequently achieved, diagnostic and control systems have been commissioned, the H-mode accessed, magnetic error fields identified and mitigated, and the first physics research campaign carried out. During ten run weeks of operation, NSTX-U surpassed NSTX record pulse-durations and toroidal fields (TF), and high-performance $~ 1 \mathrm{MA} \mathrm{H}$-mode plasmas comparable to the best of NSTX have been sustained near and slightly above the $n=1$ no-wall stability limit and with $\mathrm{H}$-mode confinement multiplier $\mathrm{H}_{98 y, 2}$ above 1 . Transport and turbulence studies in L-mode plasmas have identified the coexistence of at least two ion-gyro-scale turbulent micro-instabilities near the same radial location but propagating in opposite (i.e. ion and electron diamagnetic) directions. These modes have the characteristics of ion-temperature gradient and micro-tearing modes, respectively, and the role of these modes in contributing to thermal transport is under active investigation. The new second more tangential neutral beam injection was observed to significantly modify the stability of two types of Alfven eigenmodes. Improvements in offline disruption forecasting were made in the areas of identification of rotating MHD modes and other macroscopic instabilities using the disruption event characterization and forecasting code. Lastly, the materials analysis and particle probe was utilized on NSTX-U for the first time and enabled assessments of the correlation between boronized wall conditions and plasma performance. These and other highlights from the first run campaign of NSTX-U are described.}

Keywords: NSTX-U, spherical tokamak, Alfven eigenmodes, plasma material interactions, boronization, error fields

(Some figures may appear in colour only in the online journal)

\section{Introduction}

The National Spherical Torus Experiment (NSTX) has undergone a major upgrade to become NSTX Upgrade (NSTX$\mathrm{U})$. NSTX-U mission elements include: exploring unique Spherical Torus/Tokamak (ST) parameter regimes to advance predictive capability for ITER and beyond, developing solutions for the plasma-material interface challenges, and advancing the ST as a possible fusion nuclear science facility (FNSF) or pilot plant [1-4]. NSTX-U [5-7] has two major new tools: (1) a new central magnet and (2) a new second more tangential neutral beam injector (NBI). The new central magnet of NSTX-U will ultimately double the TF from 0.5 to $1 \mathrm{~T}$, double the plasma current from 1 to $2 \mathrm{MA}$, and quintuple the pulse duration from 1 to $5 \mathrm{~s}$ relative to NSTX. The new second NBI of NSTX-U doubles the auxiliary NBI power from 5 to $10 \mathrm{MW}$ and is projected to enable fully noninductive plasmas at the $\sim 1$ MA level.

During the past two years the Upgrade Project was completed, first plasma was achieved, diagnostic and control systems were commissioned, error fields (EFs) corrected and mitigated, the H-mode accessed (see figure 1, lower panel), and the first physics research campaign carried out. In the 20152016 research campaign, NSTX-U operated for ten run weeks and produced 1066 plasma shots. The H-mode was accessed during the first two weeks of operations after first boronization was performed and NBI was used to heat the plasma. H-mode access became routine, and more than 30 machine proposals for commissioning major capabilities and several physics experiments were carried out as described below. In June of
2016, the upper primary divertor coil (PF1AU) developed an internal short that was not repairable. This coil and several other center-stack components will be replaced or enhanced during 2017. The NSTX-U program currently has a goal of resuming plasma operation during 2018.

Results from the initial machine commissioning and plasma scenario development, and highlights from physics experiments and modeling carried out for NSTX-U are described below. Section 2 describes commissioning and L- and $\mathrm{H}$-mode plasma scenario development, section 3 describes research highlights by topical science area, including energetic particles, transport and turbulence, macroscopic stability, boundary science, and solenoid-free plasma start-up simulations with application to NSTX-U, and section 4 briefly summarizes these results.

\section{NSTX upgrade commissioning and scenario development}

Substantial facility commissioning and scenario development was accomplished in NSTX-U during the 2015-16 research campaign. All magnetic diagnostics needed for off-line and real-time equilibrium reconstructions were commissioned, and the real-time EFIT reconstructions and the ISOFLUX plasma boundary shape control algorithm were commissioned. Key profile diagnostics were commissioned including multi-point Thomson scattering and charge exchange recombination spectroscopy (CHERS). Long-pulse L-mode scenarios were developed for transport studies and intrinsic EF detection and correction. High-performance $\mathrm{H}$-mode plasmas 

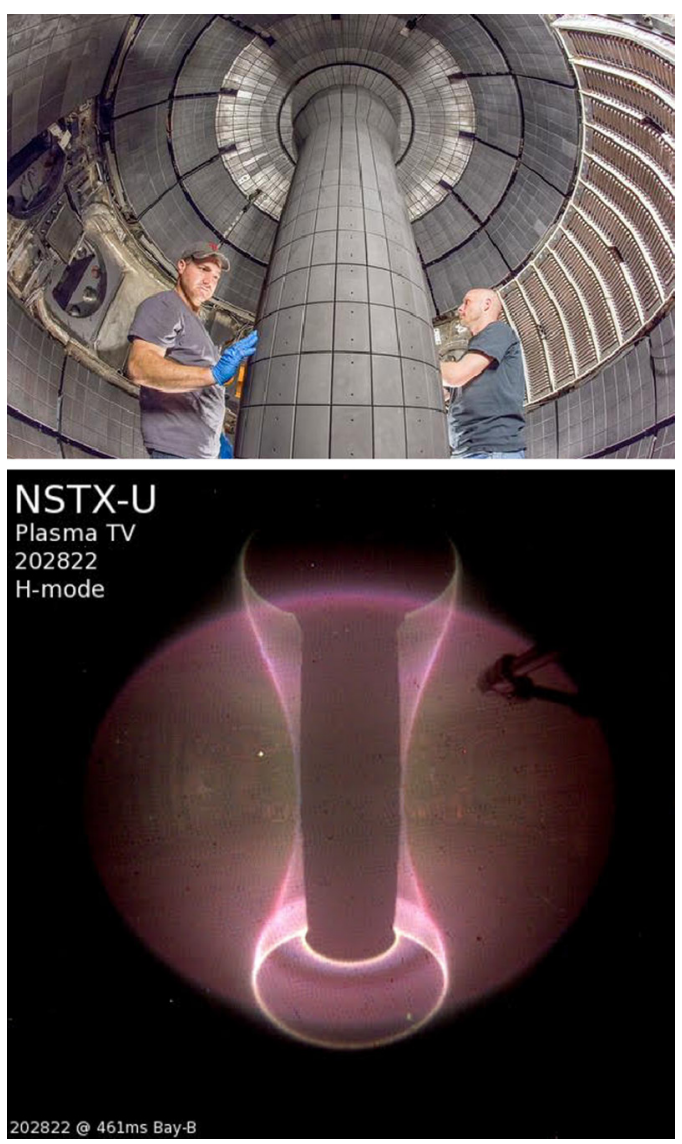

Figure 1. Top: New NSTX-U centerstack central magnet. Bottom: image of NSTX-U H-mode plasma.

operating near and slightly above the $n=1$ no-wall stability limit were also developed.

Figure 2 shows comparisons of NSTX (black) and NSTX-U (red) L-mode plasmas each heated with $1 \mathrm{MW}$ of NBI heating. The NSTX plasma had a flat-top line-average density of $2.4 \times 10^{19} \mathrm{~m}^{-3}$ versus $1.6 \times 10^{19} \mathrm{~m}^{-3}$ for the NSTX-U plasma, and the $T_{\mathrm{e}}(0)$ values for the NSTX and NSTX-U plasmas were $0.7-0.8 \mathrm{keV}$ and $1.4-1.5 \mathrm{keV}$, respectively. Both the NSTX and NSTX-U plasmas reach their respective ohmic solenoid current limits at the end of the plasma current flattop. The significantly larger (factor of 3 ) ohmic solenoid flux available in NSTX-U combined with higher $T_{\mathrm{e}}$ and $50 \%$ higher $\mathrm{TF}$ at fixed major radius resulted in a factor of 5 increase in L-mode flat-top pulse duration. For reference, the vacuum TF at the plasma geometric centers for the NSTX $\left(R_{0}=0.86\right.$ $\mathrm{m})$ and NSTX-U $\left(R_{0}=0.94 \mathrm{~m}\right)$ plasmas are $0.44 \mathrm{~T}$ and 0.63 $\mathrm{T}$, respectively. Reproducible saw-toothing plasmas were achieved in NSTX-U for the first time, and this enabled new studies of tearing-mode stability and triggering not previously accessible in NSTX. Figure 2 shows plasma current sustained past $t=2.0 \mathrm{~s}$, and this plasma duration exceeds the record pulse duration achieved in NSTX in any confinement regime (L-more or H-mode) and any heating power. Further, figure 2 also shows a TF of $0.59 \mathrm{~T}$ (at a reference $R_{0}=1 \mathrm{~m}$ ) sustained with a flat-top exceeding $2 \mathrm{~s}$, and this TF exceeds the maximum field achievable on NSTX $\left(0.55 / 0.48 \mathrm{~T}\right.$ at $R_{0}=0.86 / 1.0$ $\mathrm{m})$ for a factor three times longer than in NSTX. Thus, in a
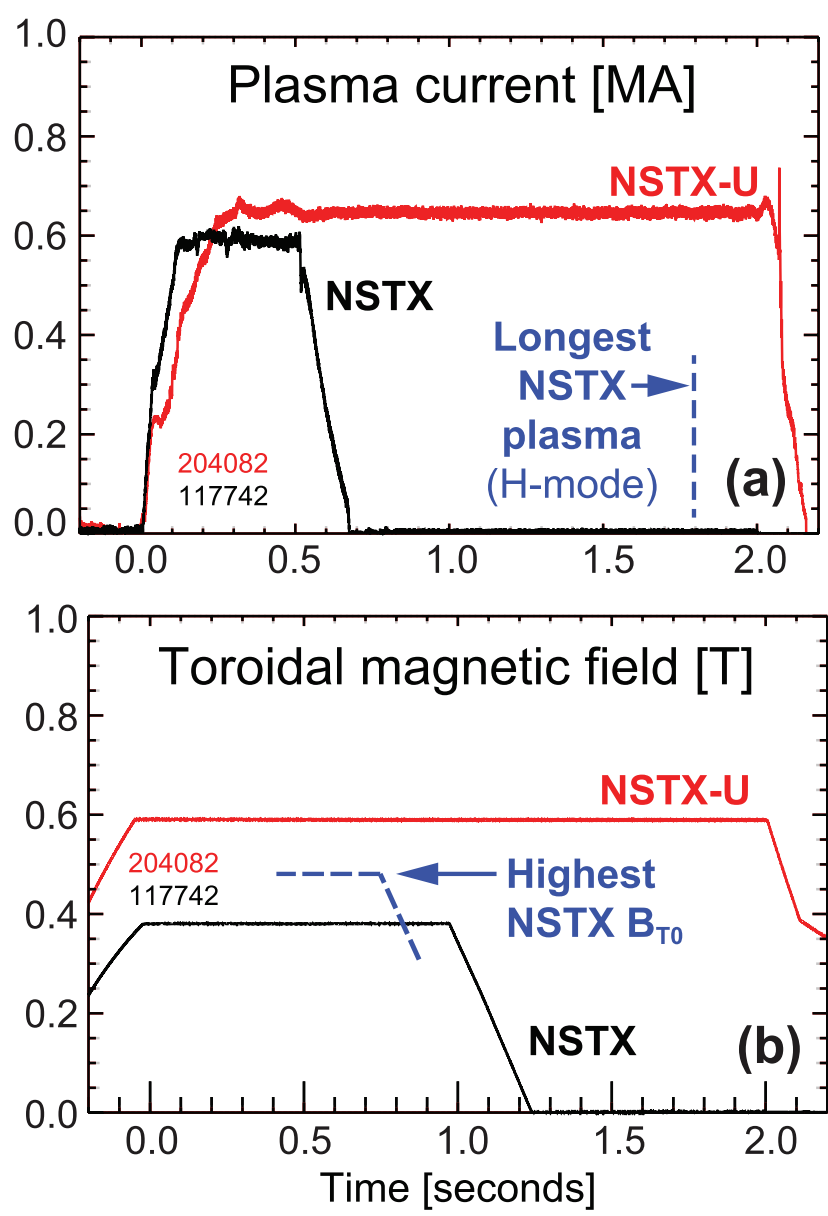

Figure 2. Comparison of NSTX and NSTX-U L-modes: (a) plasma current and $(b)$ vacuum TF at reference $R_{0}=1 \mathrm{~m}$.

single plasma discharge, figure 2 shows TF and flat-top and plasma pulse duration simultaneously, exceeding the highest values achieved in NSTX [8]. The TF shown in figure 2 also represents the maximum TF used during the first NSTX-U run campaign.

While H-mode access was obtained rapidly in NSTX-U (within the first two weeks of post-bake-out operation), longpulse H-mode operation required significantly more development than the relatively rapid L-mode development described above. Critical elements of MHD-stable long-pulse H-mode operation with boronization on NSTX and NSTX-U include sufficient heating power to sustain regular ELMs, adequate EF correction, and the utilization of early H-mode access, i.e. $\mathrm{H}$-mode access during the plasma current ramp-up [9].

Such early H-mode access plays an important role in increasing the plasma temperature and the off-axis bootstrap current-both of which slow the current penetration, significantly lower the plasma internal inductance $l_{\mathrm{i}}$ [10], allow increased early elongation, and help maintain an elevated safety factor profile in the plasma core. As shown in figure 3(a), the achieved flat-top elongation at the time of maximum stored energy in NSTX-U approaches or matches values achieved in NSTX for both higher inductance $\left(l_{\mathrm{i}} \geqslant 1\right)$ L-modes and intermediate inductance $\left(l_{\mathrm{i}} \geqslant 0.7-1\right) \mathrm{H}$-modes. Improvements to the plasma vertical position detection for NSTX-U have facilitated access to comparable maximum elongation $\kappa$ values 

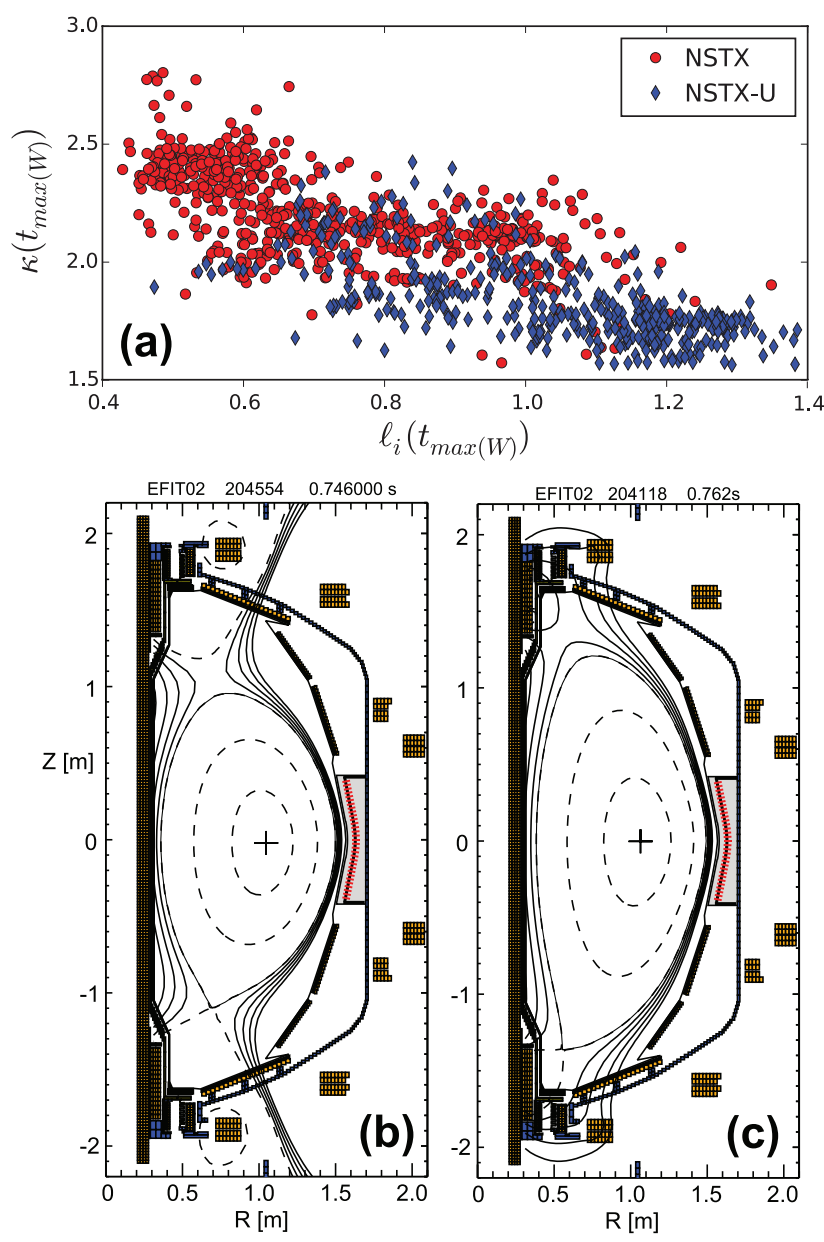

Figure 3. (a) Comparison of NSTX and NSTX-U elongation $\kappa$ versus internal inductance $l_{\mathrm{i}}$, equilibrium reconstruction of poloidal flux contours for NSTX-U for $(b) 900 \mathrm{kA} \mathrm{L}$-mode with $l_{\mathrm{i}}=1.05$, $\kappa=1.8$, and $(c) 1 \mathrm{MA}$ H-mode with $l_{\mathrm{i}}=0.65, \kappa=2.32$.

despite the increased aspect ratio of NSTX-U relative to NSTX. Figures 3(b) and (c) show equilibrium reconstructions of representative L-mode and $\mathrm{H}$-mode plasmas. As $\mathrm{H}$-mode was accessed progressively earlier, lower $l_{\mathrm{i}}$ values could be sustained and higher $\kappa$ stably achieved. However, while $\kappa$ up to 2.3-2.4 was achieved at $l_{\mathrm{i}} \sim 0.7$ in NSTX-U and $l_{\mathrm{i}}$ values as low as $0.5-0.55$ were beginning to be accessed, insufficient run time was available (due to the divertor PF coil failure) to optimize the ramp-up and increase $\kappa$ above 2.0 at the lowest $l_{\mathrm{i}}$ values. Accessing earlier $\mathrm{H}$-mode and high $\kappa=2.5-2.8$ at $l_{\mathrm{i}}<0.6$ is high-priority for future NSTX-U run campaigns since for both NSTX and NSTX-U high- $I_{\mathrm{P}}$ scenarios, lack of early $\mathrm{H}$-mode access often resulted in triggering $m / n=2 / 1$ tearing modes that could slow and lock if intrinsic EFs were too high.

Figure 4 shows a sample of the progression of $\mathrm{H}$-mode plasma scenarios obtained in NSTX-U. As is evident in figure 4, the early $\mathrm{H}$-mode plasma shot 204118 sustained a plasma current flat-top of $1 \mathrm{MA}$ lasting until $t=1.25 \mathrm{~s}$ which matches the best-performance 1 MA plasmas obtained in NSTX. The maximum plasma current and length of discharge steadily increased through the run as the available neutral beam power and reliability increased and earlier $\mathrm{H}$-mode
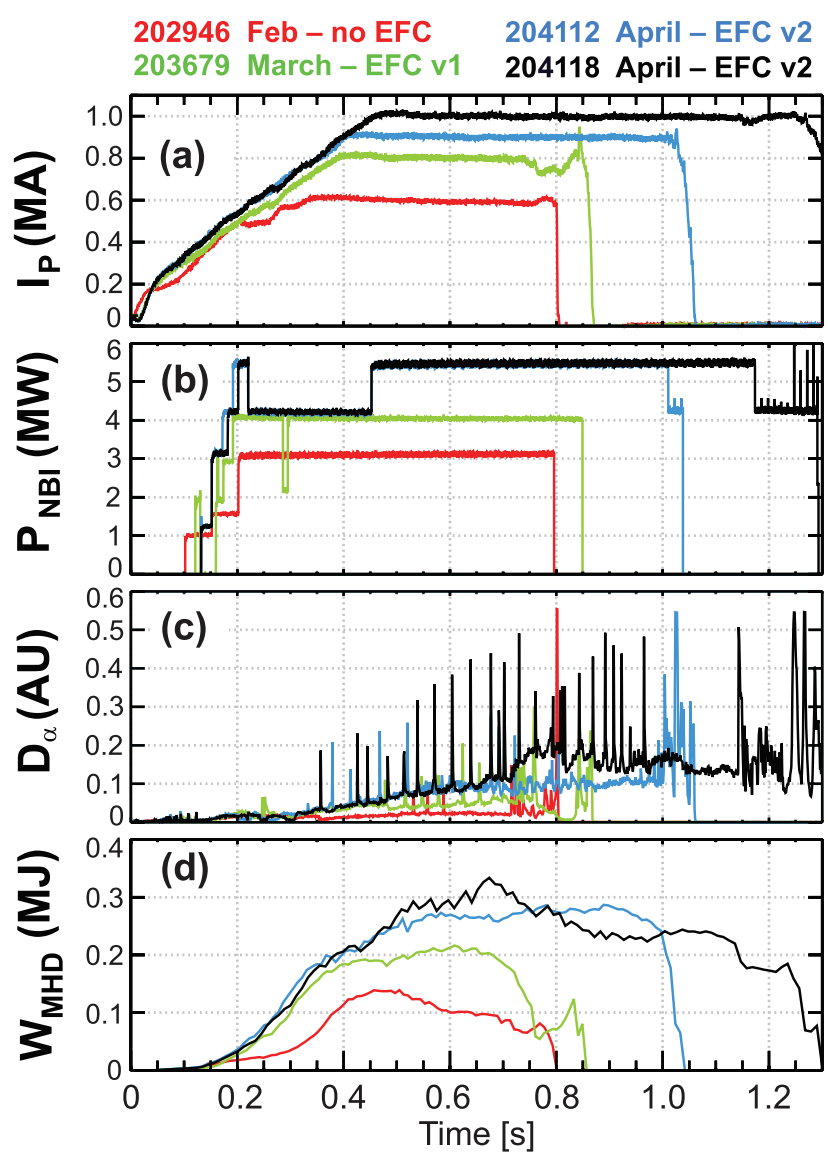

Figure 4. (a) Plasma current, (b) NBI power, (c) ELM behavior, and $(d)$ stored energy at different stages of EF correction and H-mode development.

access was achieved by improvements in the control of vertical position, $X$-point location, and the inner gap evolution which facilitated earlier achievement of diverted plasmas with higher elongation during both the current ramp-up and flattop. Progressively improved correction of EFs as described in section 3.3 also assisted in the avoidance of deleterious MHD instabilities. For example, the stored energy decrease in shot 204118 starting near $t=0.7 \mathrm{~s}$ as shown in figure $4(d)$ is due to the onset of a core $n=1$ mode which would otherwise lock and disrupt the plasma with prior less-optimized EF correction.

Figure 5 shows that NSTX-U has re-established flat-top periods (yellow band) with weak/no low- $n$ modes, ITER $\mathrm{H}$-mode confinement enhancement factor $\mathrm{H}_{98 y, 2}>1$, and operating at or above the estimated $n=1$ kink no-wall stability limit [11] even without active $n=1$ feedback control. Such plasma scenarios are very suitable for follow-on studies of H-mode transport and stability. Future experiments will advance the neutral beam heating, plasma shape control, and EF correction to the levels needed to increase the NSTX-U plasma current up to 1.5-2.0 MA at an on-axis TF of 0.75-1 T.

NSTX-U also made significant progress in 2015-16 toward identifying impending disruptions and in ramping down the plasma when disruptions can no longer be avoided. Key to this progress was the implementation of a new 'Shutdown State Machine' in the plasma control system (PCS). There 


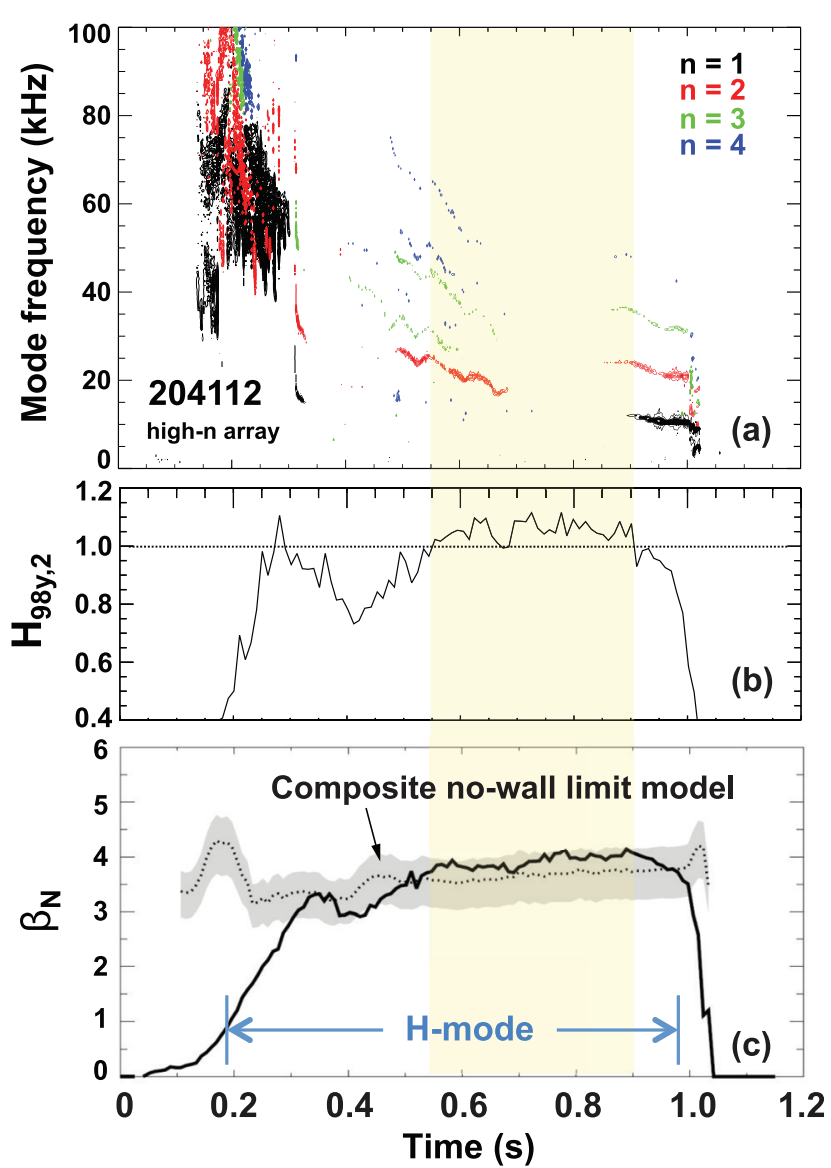

Figure 5. 0.9 MA H-mode (a) low- $n$ mode activity, $(b)$ normalized $\tau_{\mathrm{E}}$, and $(c) \beta_{\mathrm{N}}$.

are several motivations for implementing such a system. First, the largest forces on the coils and support structures in NSTX often occurred during transients while trying to control a disruption. By accepting that the plasma is disrupting and attempting instead to control it rapidly to zero current, these transient loads can be reduced. Second, the development of plasma shutdown methods, both during normal operations and during off-normal events, is critical for the ultimate development of ITER and future high-current tokamak/ST devices where large disruption forces must be reduced or mitigated.

This shutdown code is based on the 'state machine' formalism as shown in figure 6. In this system, the plasma is initiated in the SS $=0$ state for ramp-up and flat-top control. There are two terminal states: $\mathrm{SS}=3$ occurs when the ohmic heating $(\mathrm{OH})$ solenoid current has exceeded a final threshold, which implies an imminent loss of $\mathrm{OH}$ current control and therefore plasma current control, while $\mathrm{SS}=4$ corresponds to the case where the plasma current has vanished (either due to being ramped down or a disruption). In either of these terminal states, all gas injection is stopped, the neutral beams are turned off, and all coil currents are returned to zero. In between the two terminal states and the initial $\mathrm{SS}=0$ state reside the two plasma ramp-down sequences. $\mathrm{SS}=1$ contains a slow ramp-down, which is intended to be entered when the plasma is in a normal state. Only (i) an operator waveform, (ii) the $\mathrm{OH}$ current dropping beneath an initial threshold, or (iii) the $\mathrm{OH}$ coil approaching an $I^{2} t$ limit could drive this

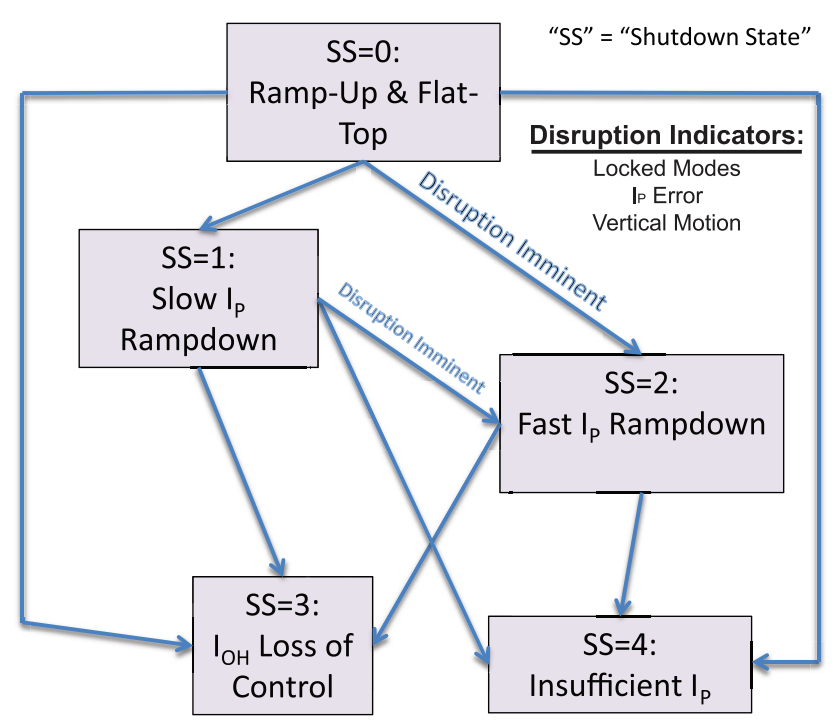

Figure 6. Present NSTX-U PCS state machine.

transition. The $\mathrm{SS}=2$ fast ramp-down state, on the other hand, is intended to cover cases where the plasma has entered an unhealthy state, and needs to be quickly ramped down. The code allows transitions to the fast ramp-down state when any of the following occur: large $n=1$ modes are detected, excessive vertical motion is detected, the fractional plasma current error exceeds a threshold, or the plasma current drops beneath a threshold.

The shutdown handling mechanism has also been used for the controlled shutdown of healthy discharges, as shown in figure 7. These plasmas are $600 \mathrm{kA}$ diverted L-modes heated with $1 \mathrm{MW}$ of neutral beam power. At $t=1.5 \mathrm{~s}$, the shutdown is initiated by a pre-programmed switch to the slow ramp-down state, and a long ramp-down of the plasma current is initiated. The NBI power transitions from steady to pre-programmed modulation. The stored energy $\left(W_{\mathrm{MHD}}\right)$ decreases at the same time due to beam power and plasma current reduction. The plasma is limited on the center column during the early phase of the ramp-down, as evidenced by the drop in elongation, and the shape is held approximately constant. The density drops throughout the ramp-down, driven again by the loss of beam fueling, lack of gas fueling, and reduction in plasma current, and this enables a roughly constant Greenwald fraction through the ramp-down. The EF correction current (i.e. proportional to PF5 vertical field coil current) and phase found optimal for the plasma current flat-top was also used during the plasma current ramp-down. These ramp-downs were used on the majority of L-mode shots in the later part of the run.

\section{Research highlights by topical science area}

\subsection{Energetic particles}

The new and more tangential second NBI of NSTX-U has already rapidly yielded important new results for Alfven eigenmode [12] stability. In particular, it was found early in the 2015-16 run that the original NSTX beam sources, with tangency radii inside the magnetic axis, would excite a similar 


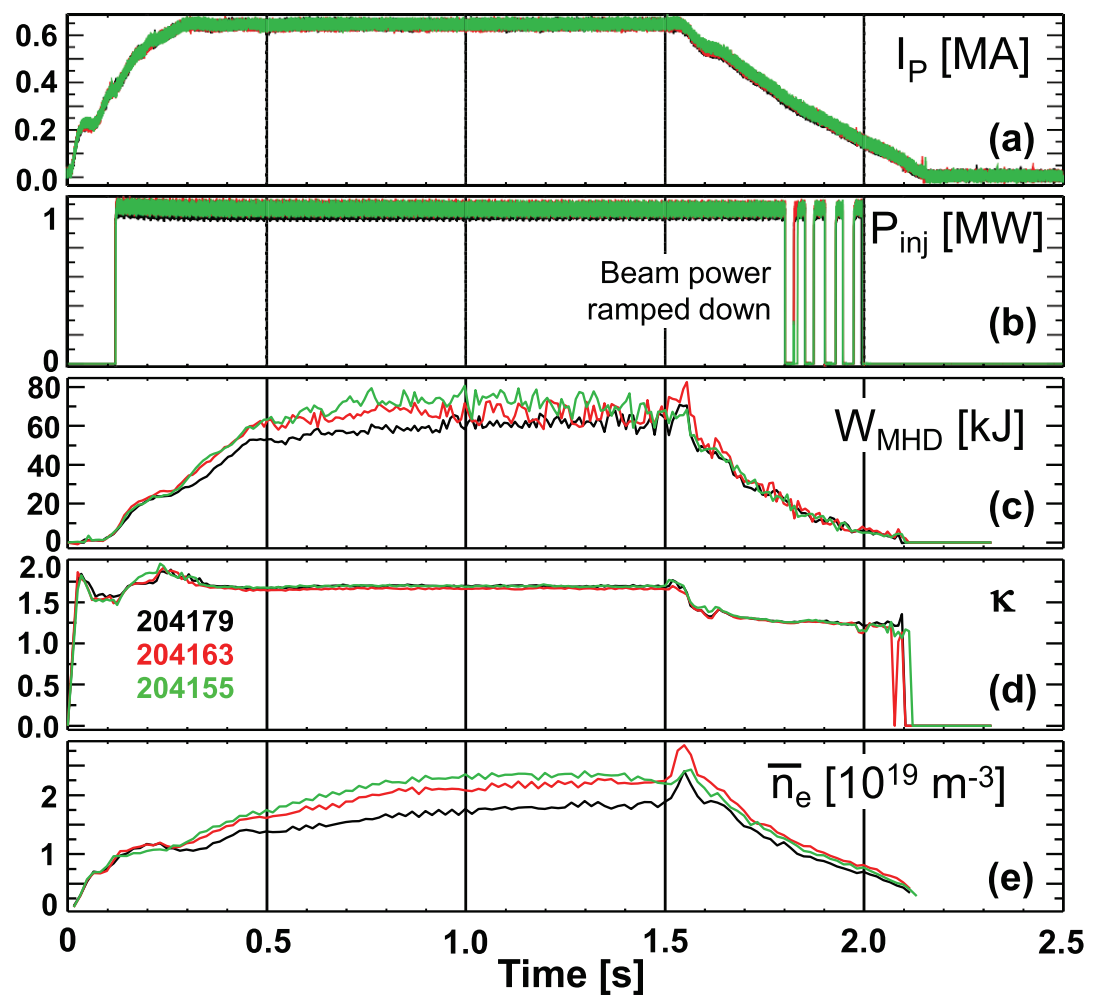

Figure 7. Comparison of three fiducial discharges from consecutive run-days showing controlled ramp-down of $(a)$ plasma current, (b) NBI power, $(c)$ stored energy, $(d)$ elongation, and $(e)$ line-average electron density.

spectrum of instabilities to those commonly seen on NSTX $[13,14]$. For the NSTX-U discharges discussed here, the nominal TF was $0.64 \mathrm{~T}$ (at $R_{0}=0.92 \mathrm{~m}$ ) which is higher than NSTX could achieve. As the new NBI line 2 sources became operational, it was quickly noted that use of these sources was anti-correlated with the presence of global Alfven eigenmodes (GAE) [15]. Further, when any outboard NBI source was added to one or more inboard NBI sources it was observed that the addition of more beam power could completely suppress the counter-propagating GAE, as shown in figure 8 . This observation is consistent with theory for GAE instability where the drive and damping of resonant fast ions was dependent on the Larmor radius and changes in the fast-ion distribution function [16].

The HYM code [14] has been used to model GAE stability, and figure 9 shows HYM simulations of the data shown in figure 8. Figure 9(a) shows the predicted most unstable modes are $n=9-11$, consistent with the observed mode numbers shown in figure $8(a)$. The measured and modelled mode frequencies are also consistent as shown in figure $9(b)$. Finally, figure $9(c)$ shows that HYM indeed predicts mode growth when the outboard NBI power is absent and mode damping when the outboard NBI power is present-both consistent with experiment. In particular, HYM simulations using the TRANSP [17] fast-ion distribution functions which include one of the new beam sources suggest that stabilization may be due to a change in the slope of distribution function at high energy and $v_{\|} / v$ combined with a net stabilizing effect from tangentially injected (i.e. smaller Larmor radius) fast ions with $k_{\perp} r_{\text {Larmor }}<1.9[16,18]$. The capability to suppress the GAE with the substitution of sources at the same neutral beam

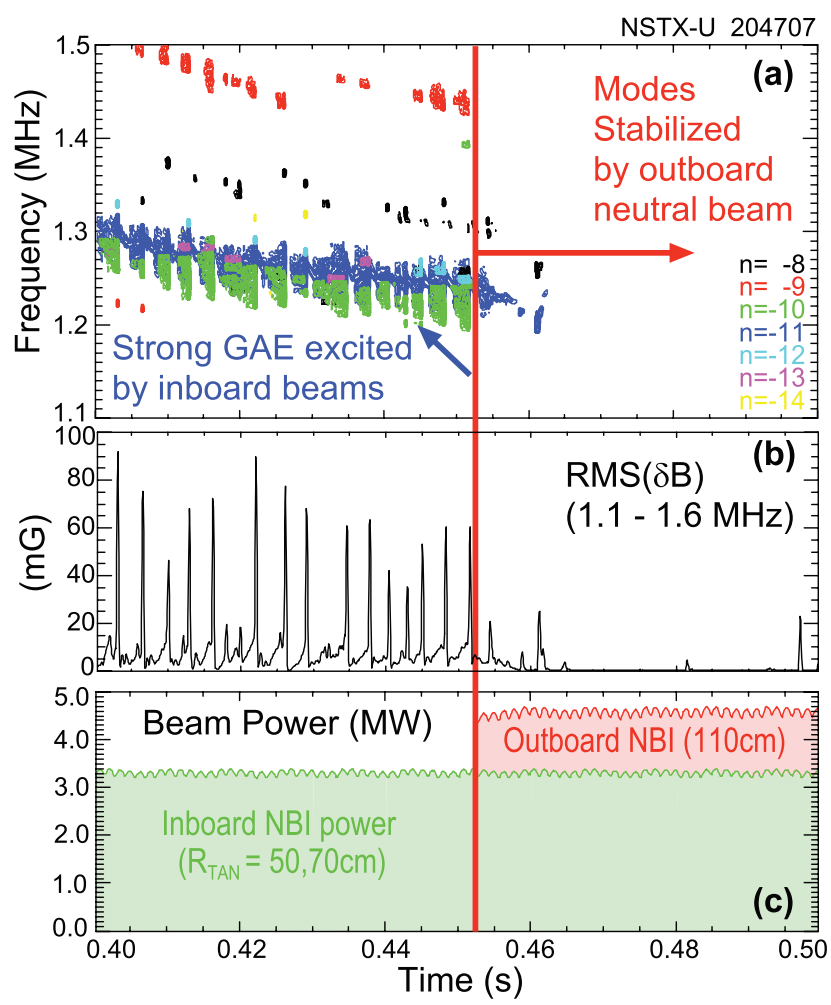

Figure 8. (a) Spectrogram showing GAE modes, (b) root-meansquare (RMS) fluctuation level of GAE, and $(c)$ beam power: total inboard source power (green), outboard source power (red).

power, or by adding more neutral beam power, will prove to be a useful tool for understanding the role of GAE instabilities in the transport of both energetic ions and thermal electrons [19]. 
HYM \#204707 t=0.44s

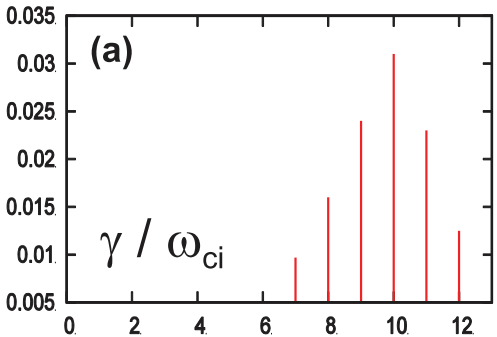

Toroidal mode number $n$

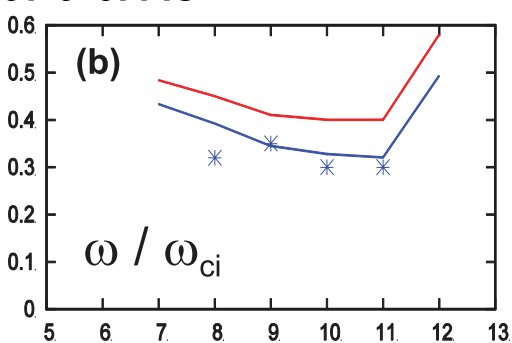

Toroidal mode number $\mathrm{n}$
HYM \#204707

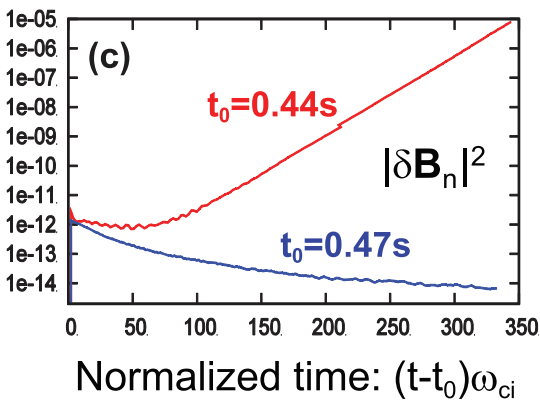

Figure 9. (a) Normalized growth rates and $(b)$ frequencies of unstable counter-GAEs from HYM simulations for $t=0.44 \mathrm{~s}$. The blue line is Doppler-shift-corrected $\omega$, stars are experimental values. (c) Evolution of magnetic energy of $n=10$ GAE from HYM near $t=0.44 \mathrm{~s}$ (red) and $t=0.47 \mathrm{~s}$ (blue).
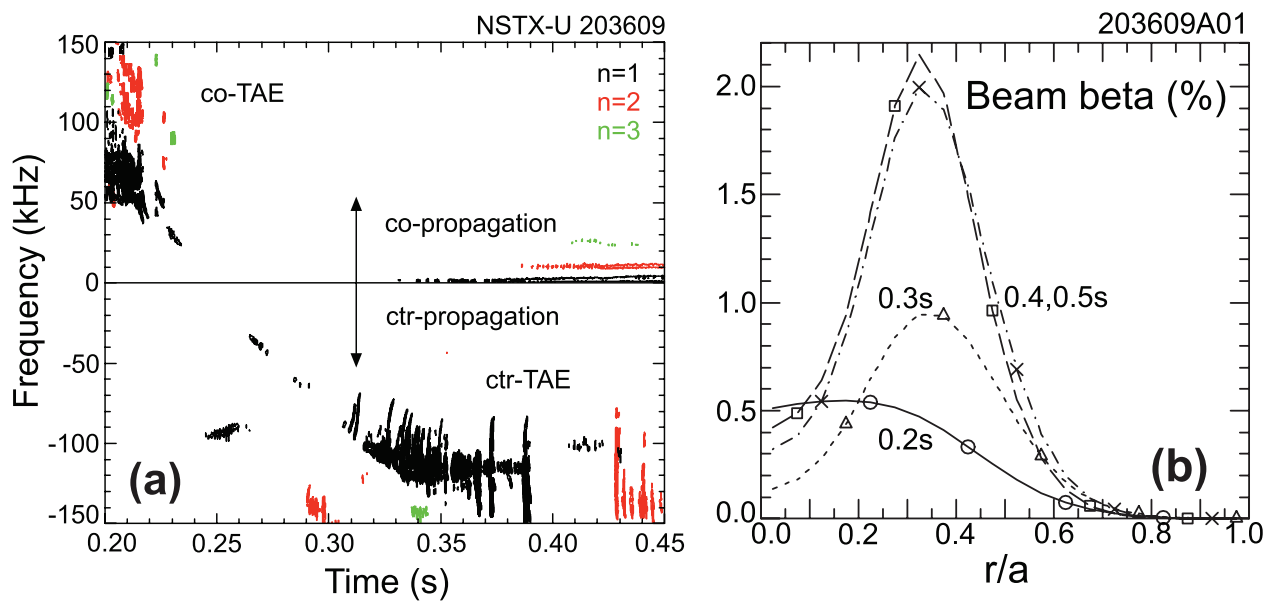

Figure 10. (a) $n=1$ TAE frequency versus time early in a plasma discharge with off-axis current drive from the most tangential NBI source of NSTX-U. (b) Fast-ion beta profile versus time as predicted by TRANSP.

Beyond substantially modifying the stability of GAE modes, the more tangential NBI sources of NSTX-U are also observed to substantially modify the stability of toroidal Alfven eigenmodes (TAEs) [12, 20, 21]. In particular, the most tangential second NBI source $\left(R_{\text {TAN }}=130 \mathrm{~cm}\right)$ is observed to generate counter-propagating TAEs. Figure 10(a) shows that the TAE frequency is initially positive/co-propagating near $t=0.2 \mathrm{~s}$, but the frequency sweeps down and crosses through zero near $t=0.25 \mathrm{~s}$ and then becomes negative. The mode amplitude then becomes larger and the mode exhibits frequency chirping after $t=0.3 \mathrm{~s}$. For this shot, only the $R_{\text {TAN }}=130 \mathrm{~cm}$ source is used from $t=0.12$ to $0.7 \mathrm{~s}$ and the plasma current increases from $350 \mathrm{kA}$ at $t=0.12 \mathrm{~s}$ to a flat-top value of $800 \mathrm{kA}$ starting at $t=0.385 \mathrm{~s}$. The sign of the TAE frequency has previously been predicted to depend on the sign of the local fast-ion beta gradient [22], and figure 10(b) shows that the sign of the fastion beta gradient predicted by TRANSP changes sign inside of $r / a \approx 0.3$ consistent with theory. Taken together, the sign change of the TAE frequency and TRANSP prediction of the hollow fast-ion beta profile represent an important validation of TAE theory and also provide the first empirical evidence that the second NBI of NSTX-U can provide off-axis beam deposition which could provide a useful tool for proposed current and rotation profile control [5, 6, 23-25].
For the results described in this paper, approximately 700 NSTX-U discharges used NBI heating, 290 used some combination of the first and second NBI during a pulse, and only nine shots used only second NBI sources during a pulse. After an intensive commissioning effort, the second NBI sources ultimately achieved maximum injected powers of $1.86 / 1.96 / 2.2 \mathrm{MW}$ for $R_{\mathrm{TAN}}=110 / 120 / 130 \mathrm{~cm}$. Variations in sawtooth precursor frequency and low- $n$ tearing mode frequency were observed as the second NBI source tangency radius was varied providing qualitative evidence that the rotation and/or $q$ profile was being modified by the second NBI. However, reliable CHERS rotation and motional Stark emission pitch-angle data were not available for these scans. Systematic investigations of the impact of the first and second NBI sources on plasma performance will be carried out after a wider range of plasma scenarios has been developed and $q$-profile diagnostics become available.

\subsection{Transport and turbulence}

3.2.1. Confinement studies. During initial NSTX-U operation, parametric scans in L-mode plasmas were initiated. Confinement and transport analyses for both L- and H-modes produced in NSTX-U were carried out using the TRANSP 

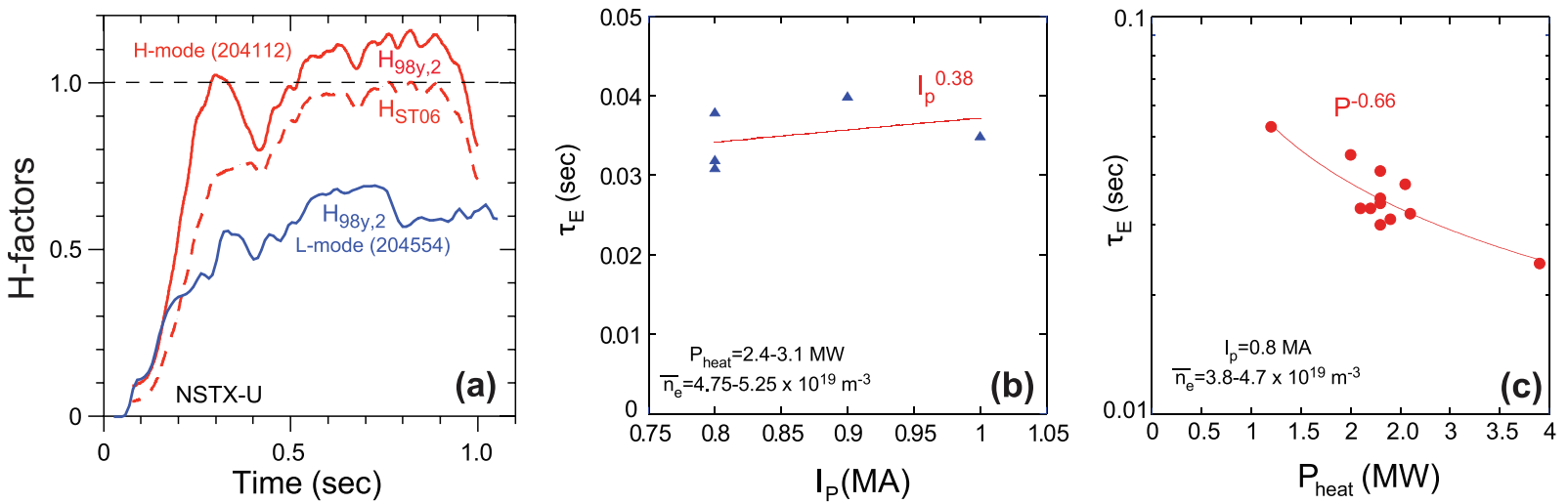

Figure 11. (a) Confinement enhancement factors versus time for H-mode and L-mode NSTX-U discharges, $(b)$ L-mode confinement scaling versus plasma current, and $(c)$ L-mode confinement scaling versus heating power.

code. This initial analysis of the data relies on imperfect input for $Z_{\text {eff }}$ and neutral density at the boundary. An assumption for $Z_{\text {eff }}$ is necessary because of low signal in the CHERS diagnostic at low beam power, as well as lack of available background emission measurements when the second neutral beam was utilized. For the results presented here, a flat $Z_{\text {eff }}=2$ profile was assumed. This value was chosen based on some available CHERS data, and there is some data from fastion D-alpha (FIDA) diagnostic and TRANSP modelling that suggest the value could be higher.

TRANSP analysis incorporated measured electron and ion kinetic profile data, magnetic equilibria as computed by kinetic EFIT, and functions of time such as plasma current, neutron production rate, toroidal magnetic field, etc. The fast ion contribution is modelled using the NUBEAM module in TRANSP. New in this calculation is the use of a feedback algorithm that adjusts the anomalous fast ion diffusivity during the calculation in order to bring the calculated and measured neutron rates into agreement. It was found, especially for lower density discharges and discharges with obvious MHD activity, that up to $50 \%$ of the fast ion density/power could be lost through shine-thru, orbits leaving the main plasma and intersecting material surfaces, or charge-exchange with thermal neutrals. Further validation of NUBEAM results against FIDA and solid-state neutral particle analyzer measurements is the subject of future work.

Figure 11(a) shows that H-mode plasmas have been produced in NSTX-U with thermal energy confinement times at the ITER ELMy H-mode scaling level or greater with $\mathrm{H}_{98 y, 2}$ up to 1.1-1.15. For comparison, L-mode plasmas have $\mathrm{H}_{98 y, 2} \leqslant 0.7$ as shown in figure $11(a)$. The H-mode plasma electron thermal diffusivity was approximately a factor of two to three lower than in L-mode discharges. Figure 11(a) also shows that NSTX-U H-mode energy confinement is so far consistent with 'ST confinement' since $H_{\mathrm{ST} 06} \approx 1$ relative to the OLSR version of the NSTX confinement scaling $\tau_{\mathrm{E}, \mathrm{th}} \propto I_{\mathrm{P}}^{0.57} B_{\mathrm{T}}^{1.08} n_{\mathrm{e}}^{0.44} P^{-0.73}[6,26]$. However, no controlled parametric scans have yet been conducted for H-mode plasmas. It is also noted that any $Z_{\text {eff }}$ uncertainty has relatively weak direct impact on the $\mathrm{H}$-factor. In particular, while $Z_{\text {eff }}$ variations could impact the beam heating, such effects are offset by adjustments in the anomalous fast-ion diffusion and/or neutral density made to obtain agreement between the measured and predicted neutron rate in TRANSP analysis. Limited parameter variation studies of the dependence of the thermal energy confinement time were possible only with L-mode plasmas where initial controlled scans were conducted. The L-mode parametric scans consisted of changing plasma current at fixed heating power and line averaged density (see figure 11(b)), and changing heating power at fixed plasma current and line averaged density (see figure 11(c)). At nearly fixed heating power in the range from $P_{\text {heat }}=2.4-3.1 \mathrm{MW}$ and line averaged densities from 4.75 to $5.25 \times 10^{19} \mathrm{~m}^{-3}$, a plasma current scan from 0.8 to $1.0 \mathrm{MA}$ was performed. No strong current dependence emerged from this scan. There is a slightly positive dependence of thermal confinement time on current $\left(I_{\mathrm{P}}^{0.38}\right)$, which is weaker than is found in conventional aspect ratio L-mode studies, but which also has a high statistical uncertainty. The power dependence of the L-mode thermal confinement time was taken from a scan of discharges with $I_{\mathrm{P}}=0.8 \mathrm{MA}, B_{\mathrm{T} 0}=0.64 \mathrm{~T}$ at $R_{0}=0.92 \mathrm{~m}$ and line averaged densities within the range of $3.8-4.7 \times 10^{19} \mathrm{~m}^{-3}$. The range of neutral beam plus $\mathrm{OH}$ power is approximately $1.1-$ 3.9 MW. There is a clear clustering of discharges in the 2-2.5 MW range, and the fit through the points is highly leveraged by single minimum and maximum power points. With these caveats this small set of discharges shows a power degradation of $P^{-2 / 3}$ (see figure $11(c)$ ) consistent with previous non-ST L-mode results [27].

The local transport in selected L-mode discharges has been assessed through local power balance calculations in TRANSP, and the results indicate that the electron thermal diffusivity is very high and anomalous, $\sim 7-20 \mathrm{~m}^{2} \mathrm{~s}^{-1}$ in the outer half of the plasma, consistent with previous NSTX results and indicating that the electron channel dominates the energy loss [28]. Ion transport is lower with $\chi_{i} \sim 1-5 \mathrm{~m}^{2} \mathrm{~s}^{-1}$ in the outer portion of the plasma and is at or above the neoclassical level there. Preliminary predictive calculations find that the Rebut-Lallia-Watkins (RLW) model for micro-tearing-induced transport [29, 30] generates electron temperature profiles in agreement with measured profiles in at least one of the L-mode discharges as shown in figure 12. The RLW model was also shown to predict $T_{\mathrm{e}}$ profiles in high collisionality NSTX H-mode discharges [31] consistent with gyrokinetic results showing the dominance of this low- $k$ mode. 


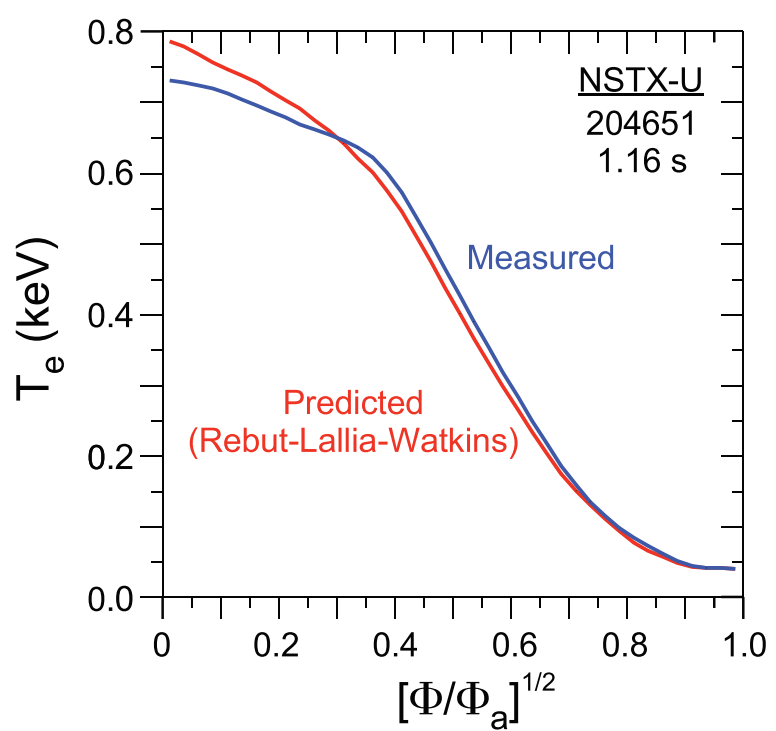

Figure 12. The measured electron temperature profile (red) versus that predicted by the RLW micro-tearing-induced electron transport model in an NSTX-U L-mode.

For the NSTX-U L-mode, gyrokinetic simulations show that micro-tearing is present but is limited in space as the dominant mode. Linear gyrokinetic studies indicate that both ion-temperature-gradient (ITG) modes (at low $k_{\theta} \rho_{\mathrm{s}}<1$ ) and ETG modes (high $k_{\theta} \rho_{\mathrm{s}}>1$ ) are predicted to be the dominant micro-instabilities outside the mid-radius, $r / a=0.5-0.8$. Micro-tearing is predicted to be dominant at low $k_{\theta} \rho_{\mathrm{s}}$ inside $r / a=0.5$. These linear results, however, do not reveal the strength of the subdominant instabilities, and it is conceivable that micro-tearing is unstable even where the ITG mode is dominant. Additional and more in depth gyro-kinetic studies are needed to better assess the role of micro-tearing in these discharges.

3.2.2. Initial turbulence measurements and simulations. Turbulence studies were also initiated during 2015-16 operations using the beam emission spectroscopy (BES) system provided by the University of Wisconsin. The BES system [32] has been used to measure ion scale turbulence fluctuations in a number of NSTX-U L-mode plasmas. Figure 13 shows the power spectra of the normalized density fluctuations (assumed to be proportional to the BES intensity, $\delta n / n \sim \delta I / I)$ in a $2.6 \mathrm{MW} \mathrm{L-mode.} \mathrm{The} \mathrm{spectra} \mathrm{are} \mathrm{mea-}$ sured at five adjacent radial positions between $\sim 135-147 \mathrm{~cm}$ (corresponding to normalized radii $r / a \sim 0.7-0.95-$ see figure $14(a)$ ) and illustrate broadband frequency fluctuations up to $\sim 200 \mathrm{kHz}$. The low frequency $(<50 \mathrm{kHz})$ portion of the spectrum may be affected by common-mode beam fluctuations. The strength, frequency-integrated over $2-200 \mathrm{kHz}$, is quite substantial increasing from $\sim 1 \%$ at the inner channel to $>4 \%$ at the outer channel, suggesting the presence of strong ion scale turbulence [33].

Bimodal turbulence has also been observed in the same L-modes using the 48 channel BES configuration as shown in figure 14(a). Figure 14(b) shows higher channel-to-channel coherence (above the statistically significant level) for
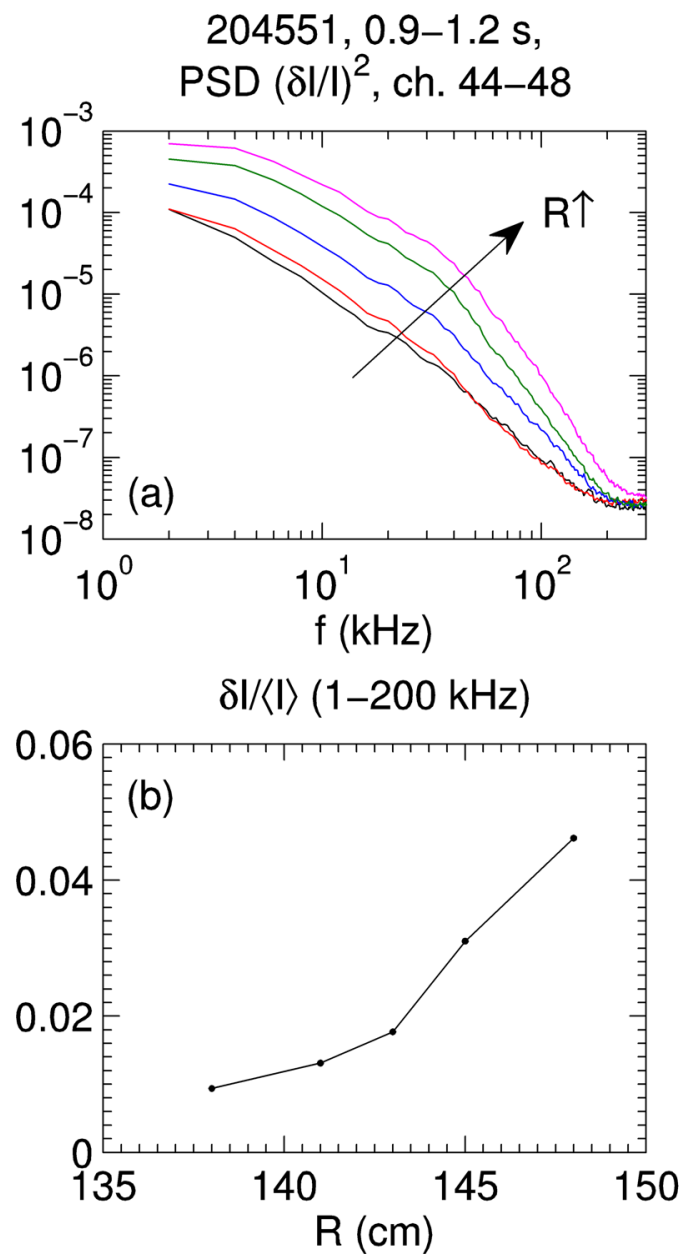

Figure 13. (a) Power spectra of normalized density fluctuations from BES at different radii. (b) Radial profile of fluctuation amplitude $(f=2-200 \mathrm{kHz})$.

frequencies between 0 and $50 \mathrm{kHz}$ and also between 75 and $150 \mathrm{kHz}$. In the lower frequency range turbulence is measured to propagate in the electron diamagnetic direction as shown in figure $14(c)$, while in the higher frequency range turbulence is measured to propagate in the ion diamagnetic direction. At ion scales $\left(k_{\theta} \rho_{\mathrm{s}}<1\right)$, linear GYRO code [34] simulations predict unstable spectra of both ITG and microtearing modes (MTM). ITG modes are predicted to be unstable for $R>135 \mathrm{~cm}$ and propagating in the ion direction. This propagation direction is consistent with BES ion modes with the caveat that Doppler shift effects need to be considered, although this shift is also in the ion direction. MTM are predicted to be unstable for $R>127 \mathrm{~cm}$ and propagating in the electron direction. It is somewhat surprising to find MTM unstable in these L-mode plasmas, however the beta is sufficiently high (4.1\%), and larger collisionality also enhances MTM destabilization.

Figure 15(a) shows that there is a strong variation in turbulence, predicted mode stability, and $E \times B$ shearing rate over a relatively narrow width $\sim 30 \rho_{\mathrm{s}}$ where $\rho^{*}=\rho_{\mathrm{s}} / a \sim 1 / 120$ and $\rho_{\mathrm{S}} / L_{\mathrm{T}} \sim 1 / 35$. This motivates the need for future global simulations of ion-scale turbulence including electromagnetic effects to treat the MTM. It is also noteworthy that there are strong local $E \times B$ shearing rates $\gamma_{\mathrm{E}}>\gamma_{\mathrm{ITG}}$ and $\gamma_{\mathrm{MTM}}$ at $R=135 \mathrm{~cm}$, but at larger major radii where $\gamma_{\mathrm{ITG}}>\gamma_{\mathrm{E}}$ the BES amplitudes 

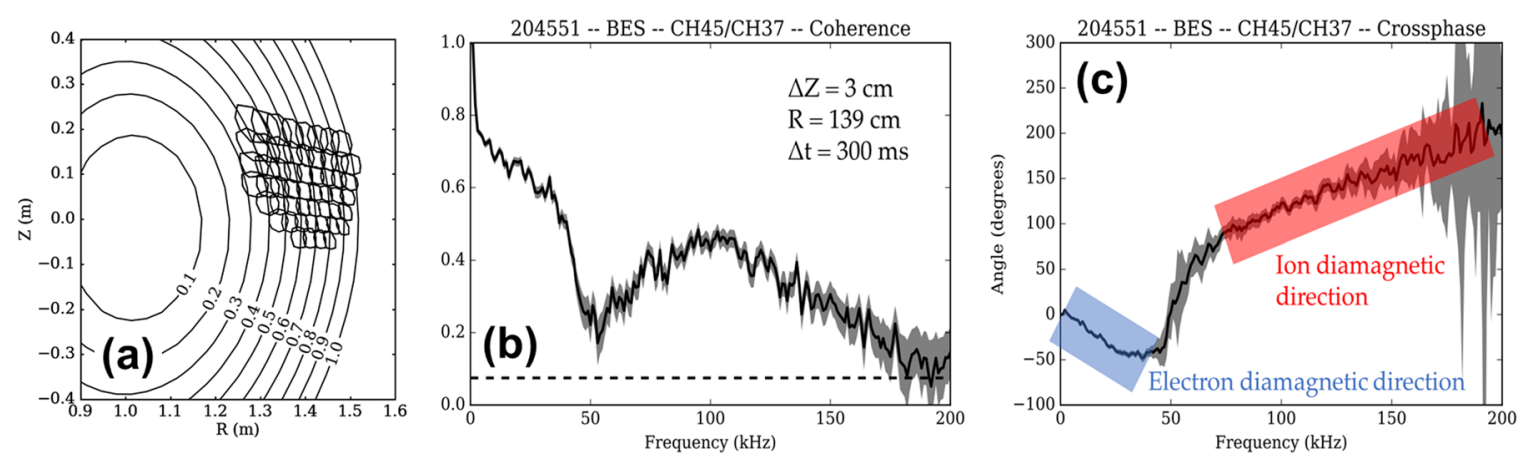

Figure 14. (a) BES channel configuration in $R, Z$ space overlaid with contours of normalized poloidal flux, $(b)$ coherence versus frequency between nearby BES channels at $R=139 \mathrm{~cm}$, and $(c)$ channel cross-phase versus frequency showing turbulence of lower/higher frequency propagating in the electron/ion diamagnetic directions, respectively.

NSTX-U 2.6MW L-mode $(204551,0.9 \mathrm{~s})$
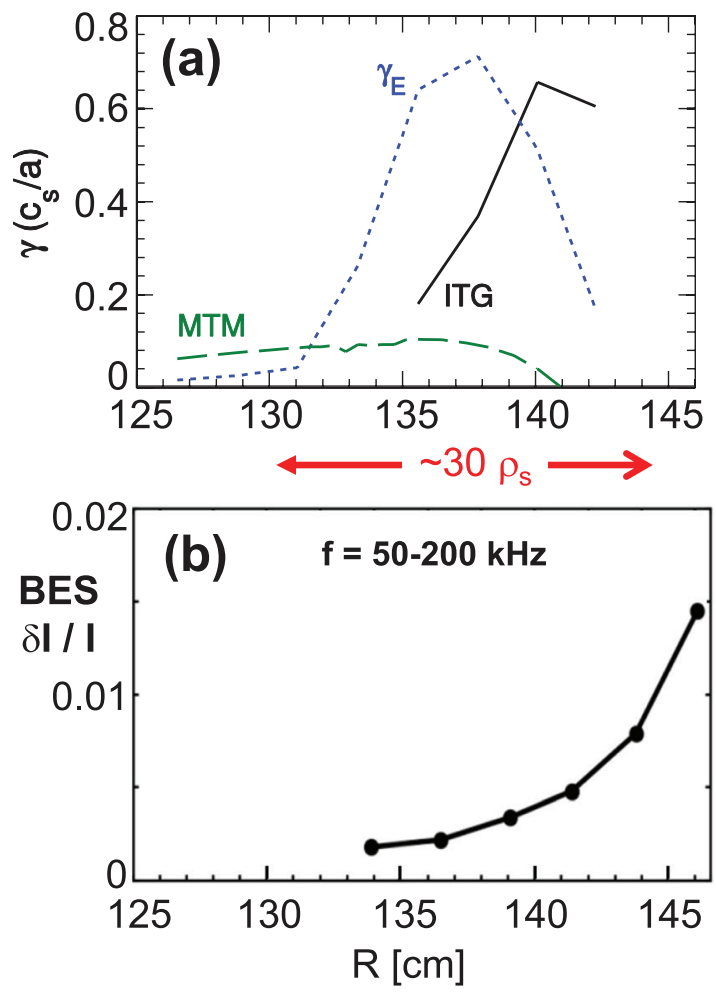

Figure 15. (a) Comparison of ITG and MTM growth-rates computed by GYRO and $E \times B$ shearing rate $\gamma_{\mathrm{E}}$. $(b)$ Measured BES relative fluctuation amplitude versus major radius in the ITG range of frequencies.

are increasing, as shown in figure $15(b)$. Thus, both the GYRO simulations and turbulence measurements are qualitatively consistent with increased ITG turbulence at larger major and minor radius. Future work will focus on comparing predicted and measured ion and electron thermal energy transport and in the $\mathrm{H}$-mode.

3.2.3. H-mode transition studies. Initial measurements of the turbulence across the L-H mode transition in NSTX-U plasmas have also been conducted using the upgraded 2D BES system. Figure 16 compares the density fluctuation spectra before and after an L-H transition at two locations. Broadband turbulence is observed up to $150 \mathrm{kHz}$ in the pedestal
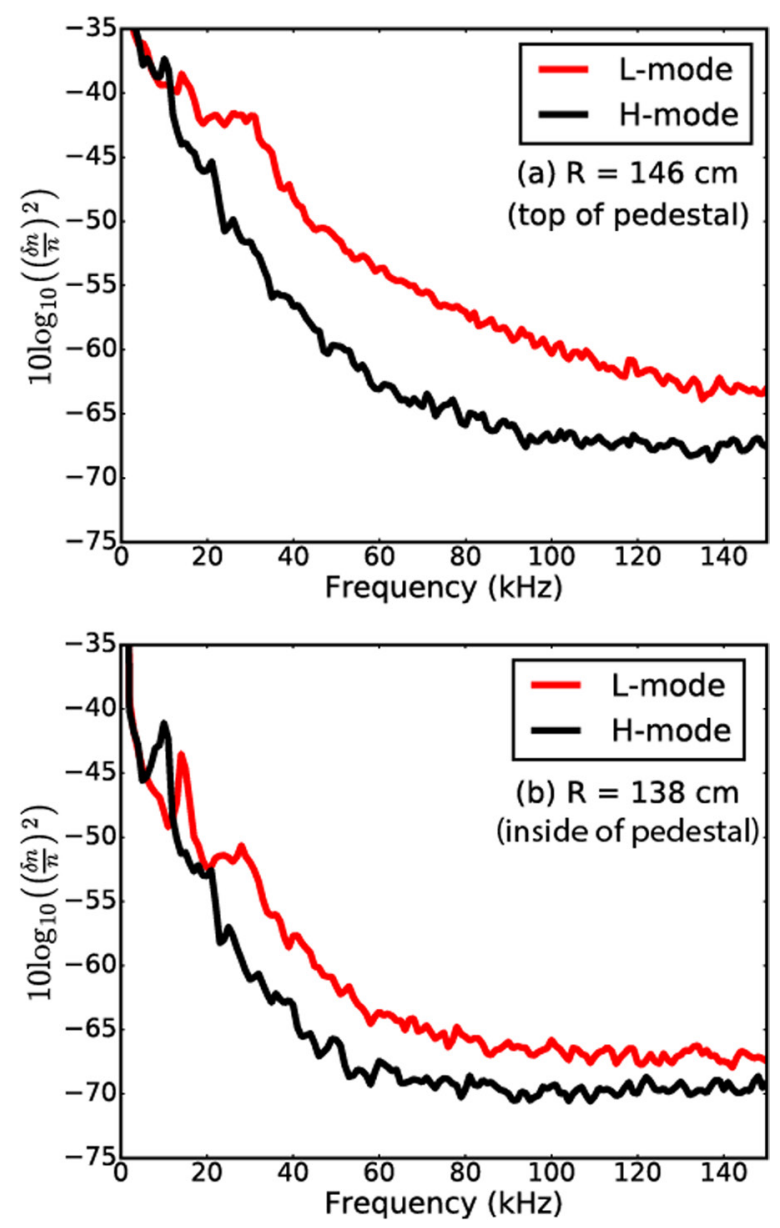

Figure 16. Auto-power spectra of normalized density fluctuations across the $\mathrm{L}-\mathrm{H}$ transition at (a) top of the pedestal and $(b) \sim 7 \mathrm{~cm}$ inward of the pedestal. Peaks in the spectrum below $15 \mathrm{kHz}$ are MHD modes. Shot 204990, $I_{\mathrm{p}}=0.65 \mathrm{MA}, P_{\mathrm{NBI}}=1 \mathrm{MW}$.

region and up to $100 \mathrm{kHz}$ several centimeters inside the pedestal. Across the L-H transition, fluctuation levels drop by a factor of six in the pedestal region and a factor of three inward of the pedestal top. These results pave the way for future studies of the turbulence across $\mathrm{L}-\mathrm{H}$ transitions.

Theoretical work to understand the dynamics of the $\mathrm{L}-\mathrm{H}$ transition [35] and the turbulence changes at the transition has recently been published [36]. The work addresses a popular model for the $\mathrm{L}-\mathrm{H}$ transition, in which the energy 
in turbulent fluctuations is directly depleted via Reynoldsstress-induced energy transfer to the zonal flows. Previous experimental attempts to validate this model used energy balance between zonal flows and non-zonal (turbulent) $E \times B$ velocities. However, the new work demonstrates that parallel electron force balance couples the non-zonal velocities with the free energy carried by the electron density fluctuations, replenishing the turbulent $E \times B$ energy until the sum of the two turbulent free energies is exhausted. Since that sum is typically two orders of magnitude larger than the energy in turbulent $E \times B$ flows alone, the Reynolds-stress induced energy-transfer mechanism is likely to be much too weak to explain the rapid turbulence suppression at the $\mathrm{L}-\mathrm{H}$ transition.

\subsection{Macroscopic stability}

3.3.1. Error fields. EFs in a machine like NSTX-U can be generated by a number of mechanical imperfections, for instance tilts of the poloidal field (PF) or TF coils or non-circularity in the PF coils. EFs were observed in NSTX, where the dominant effect was a time-dependent tilt of the TF coil as it interacted with stray fields produced by the $\mathrm{OH}$ leads [8]. For NSTX-U, this EF source was eliminated by design using co-axial leads [5]. Coil-only vacuum shots have demonstrated that this coaxial $\mathrm{OH}$ lead assembly successfully eliminates the time-dependent $n=1 \mathrm{EF}$ due to the $\mathrm{OH} / \mathrm{TF}$ interaction. However, in spite of the elimination of the $\mathrm{OH} / \mathrm{TF}$ time-dependent $\mathrm{EF}$, additional EF studies proved to be important in improving the performance of the machine. In order to better resolve the best feed-forward EF correction (EFC), $n=1$ fields were ramped linearly in time during the plasma current flat-top and with different toroidal phases (i.e. in a 'compass scan') to determine the optimum EF correction for locked mode avoidance over a sequence of shots. Very low-frequency and locked modes are detected using a low-field-side toroidal array of radial and $\mathrm{PF}$ sensors $[8,37,38]$.

The waveforms from one such compass scan are shown in figure 17, where the plasma current in figure 17( $a$ ) shows that different shots disrupt at different times. These are $700 \mathrm{kA}$ diverted L-mode plasmas formed with a $20 \mathrm{kA}$ ohmic precharge, with a density of $1.3 \times 10^{19} \mathrm{~m}^{-3}$, and heated by 1 MW of neutral beam power. Figure $17(b)$ shows the resistive wall mode (RWM)/EF coil current. At $0.7 \mathrm{~s}$, however, the RWM coil currents diverge, with ramping amplitudes of various phases, as shown in figure $17(c)$. The fact that the different phases disrupt at different applied field amplitudes confirms that a nonzero EF must be corrected in these L-mode discharges in order to provide optimum locked mode avoidance. An EF compass scan was completed for three different scenarios: (i) the case shown in figure 17, (ii) a similar case at twice the plasma density, and (iii) a comparable high density case with an $8 \mathrm{kA}$ (rather than $20 \mathrm{kA}$ ) $\mathrm{OH}$ pre-charge. The second scan, which was conducted to assess the density scaling of the locking threshold in this $1 \mathrm{MW}$ beam-heated L-mode scenario, found that the locking threshold was largely unaffected by the higher density; this implies that the
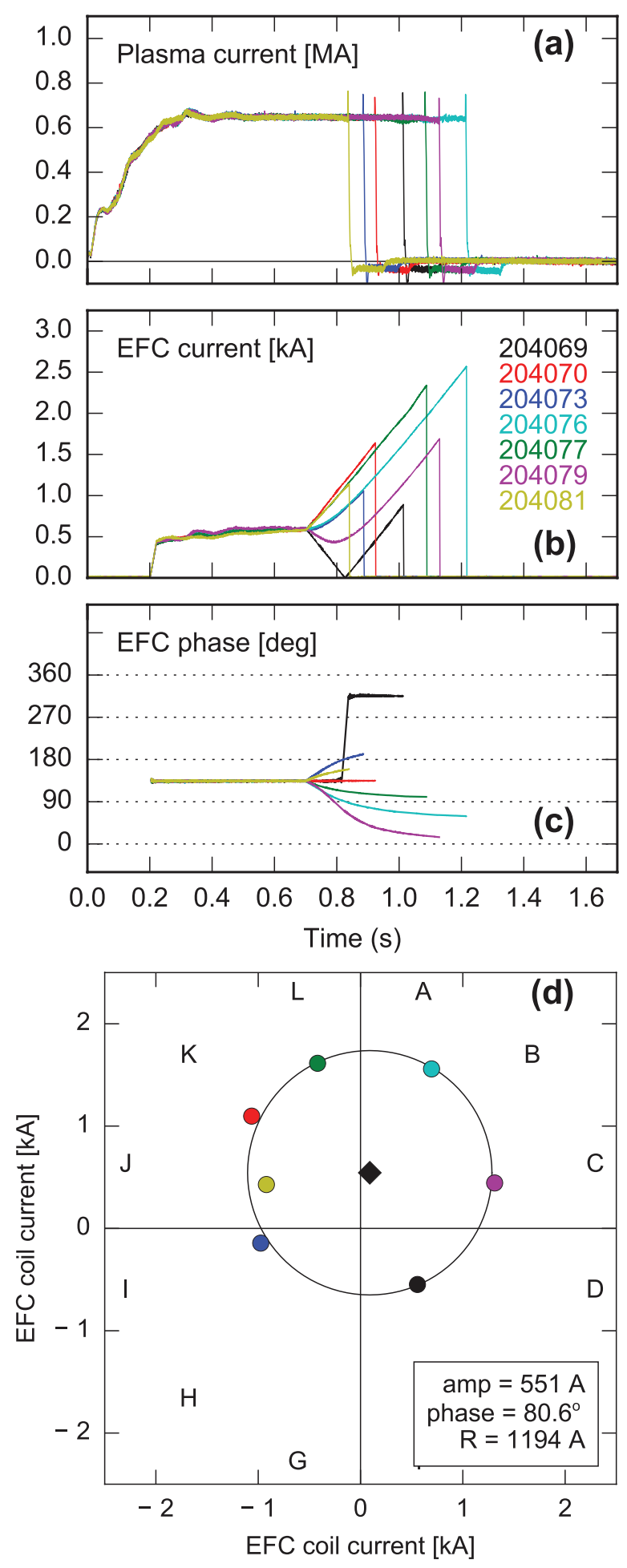

Figure 17. (a) Plasma current, (b) EFC coil currents, (c) phase of applied field from a sample compass scan, and $(d)$ locking circle showing amplitude and phase of the EF.

rotation driven by the beam is the dominant effect rather than the diamagnetic rotation that is thought to dominate in ohmic plasmas. The third and final scan, which was conducted to assess if the EF measured in the compass scans is due to the $\mathrm{OH}$ coil, found no change in the required EF correction. The fact that the required correction did not change with a large change in the $\mathrm{OH}$ pre-charge implies that the $\mathrm{OH}$ is not the 

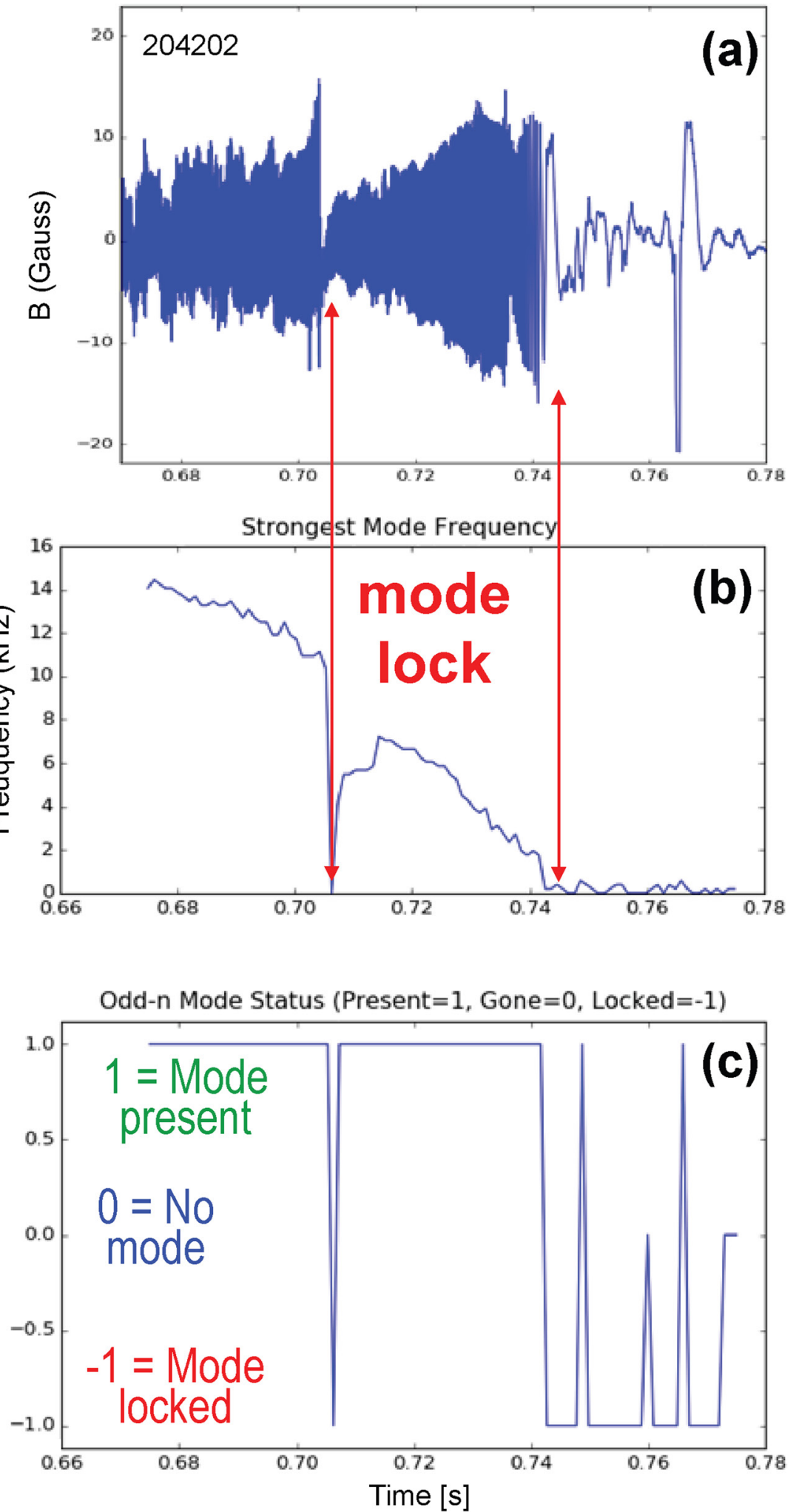

Figure 18. DECAF identification of rotating and locked odd- $n$ MHD modes showing mode $(a)$ amplitude, $(b)$ toroidal frequency, and (c) status.

source of the EF measured by the compass scans. The optimized EF correction determined from these compass scans enabled the highest performance $\mathrm{H}$-mode plasmas from the first NSTX-U run campaign-for example shots 204112 and 204118 shown in figures 4 and 5. Recent coil metrology activities indicate PF5 vertical field coil non-circularity and TF central rod tilt $\left(\sim 0.06^{\circ}\right)$ are likely the dominant sources of static $n=1 \mathrm{EF}$ and result in an $n=1 \mathrm{EF}$ magnitude of 4-6 Gauss major-radial field at the outboard plasma edge for plasma currents in the range of $1 \mathrm{MA}$. 
3.3.2. Disruption forecasting. The state machine described in section 2 has already proven to be very effective at identifying major losses of plasma confinement and/or control and ramping down the plasma. Disruption prevention and avoidance is also an important operational goal for future devices, and the disruption event characterization and forecasting (DECAF) code [39] was written at NSTX-U in order to facilitate a comprehensive framework for disruption prevention through forecasting and avoidance, or prediction and mitigation of the detrimental consequences. The ultimate goal of such an approach is to provide forecasts, which integrate with a disruption avoidance system and are utilized in real-time during a device's operation. Previously reported DECAF work focused on the first step: quantitative statistical characterization of the chains of events which most often lead to disruption of plasmas. Progress on the development of DECAF was made in three areas during the last two years: (1) identification of rotating MHD modes, (2) characterization of a set of RWM disruptions, and (3) the development of a reduced kinetic model for RWM stability [39].

An essential step for DECAF analysis of tokamak data is identification of rotating MHD activity, such as neoclassical tearing modes. The initial goals were for the code to identify existence of rotating MHD modes and to track characteristics that lead to disruption, such as rotation bifurcation and mode locking. The approach taken was to apply a fast Fourier transform analysis to determine the mode frequency and bandwidth evolution.

Figure 18(a) shows the odd- $n$ magnetic signals for NSTX-U discharge 204202. Figure 18(b) shows the mode frequencies determined by DECAF and figure 18(c) shows the mode status. As indicated by the red vertical arrows in figures 18(a) and $(b)$, this shot exhibits a transient locking event near $t=0.706 \mathrm{~s}$ and a longer-lived locking event after $t=0.74 \mathrm{~s}$. For the later locking case the mode status is more consistently declared locked by DECAF as shown in figure $18(c)$. Such real-time analysis could potentially provide much earlier warning of potential mode locking and disruption onset than is currently achievable with the low-frequency RWM/EF sensors already included in the PCS state-machine. Activities are already underway to implement a real-time rotating MHD identification algorithm for NSTX-U, and DECAF-based algorithms will ultimately be linked to the plasma shutdown handling mechanisms described in section 2 .

\subsection{Boundary science}

3.4.1. Advanced divertor simulations. Understanding of power exhaust and divertor geometry is required for supporting operational regimes in NSTX-U with acceptable heat flux on divertor targets at full plasma current and heating power. Such understanding is also important for next-step devices including FNSFs and pilot plants. While insufficient run-time was available in the initial NSTX-U run campaign to perform advanced divertor experiments, a range of simulation tools have recently been utilized to assess advanced divertor configurations for NSTX/NSTX-U [40-42] including the effects of 3D fields (e.g. for EF correction and/or resonant magnetic perturbation (RMP) ELM suppression) on plasma equilibrium response, divertor field-line topology, and plasma transport in the pedestal and scrape-off layer. The simulation tools used include UEDGE which simulates edge plasmas with neutrals and impurities, the two-fluid resistive MHD code M3D-C ${ }^{1}$ to estimate plasma responses to $3 \mathrm{D}$ perturbations, and the code EMC3-EIRENE for calculating particle, momentum, and energy transport simulations and the effect of RMPs on transport in the null-point of the snowflake divertor and related configurations.

As an example, a set of advanced divertor configurations in NSTX-U have been explored with EMC3-EIRENE and compared to a standard divertor (SD) configuration. A generalization to the classical snowflake results in a set of significantly different divertor geometries. The implementation of the magnetic configuration is very flexible in EMC3-EIRENE and makes this a useful tool for benchmarking and comparing different advanced divertor configurations. Simulation results for the edge plasma density are shown in figure 19 for $(a)$ a SD configuration, $(b)$ near exact snowflake divertor $(\mathrm{neSF}+)$ and $(c)$ an X-divertor like configuration (XD) generated by an asymmetric snowflake minus (aSF-). All simulations are based on the same input parameters for fuelling rate, heating power and anomalous cross-field diffusion. The simulated peak heat flux (see figure $19(g)$ ) is correlated to the flux expansion on the target: the neSF + configuration has a reduced flux expansion with respect to the SD configurations, which results in heat flux peaking by a factor of 3 . The aSF-/XD configuration, on the other hand, has an increased flux expansion that allows a mitigation of peak heat load by $40 \%-45 \%$. These results are qualitatively consistent with previous studies of the flux convergence and divergence for the neSF + and aSF-/XD configurations, respectively [43, 44]. It is also noted that the divertor configuration previously projected to be used for high heat flux mitigation in NSTX-U is the aSF-/XD [5]. Magnetic perturbations result in the splitting of the separatrix into two distinct branches of helical lobes that guide field lines from the bulk plasma to the divertor targets (see figures 19(d)-(f)). Another important result from these studies of non-axisymmetric fields in advanced divertors is that strong localized flux expansion is predicted to mitigate the non-axisymmetric peaking of divertor heat loads [45]. This reduction in non-axisymmetric peaking is evident in the similar heat flux profiles (within $20 \mathrm{~cm}$ of the separatrix) of the axisymmetric reference and reference + RMP cases for the aSF-/XD shown in light blue in figure $19(\mathrm{~g})$.

\subsubsection{Boronization assessment using materials analysis and} particle probe. The materials analysis and particle probe (MAPP) [46] was utilized for the first time on NSTX-U to obtain in situ and between plasma shot X-ray photoelectron spectroscopy (XPS) measurements of the chemical evolution of the plasma facing surface. MAPP is the first in vacuo surface analysis diagnostic directly integrated into a tokamak and can perform chemical surface analysis of plasma facing samples exposed in the vessel without sample retrieval from the tokamak vacuum. On NSTX-U the MAPP probe head is inserted into a gap at the outer perimeter of the lower divertor with the samples flush with the divertor tiles when exposed to plasma [47]. During the first run campaign, MAPP carried two 
Standard Divertor Near-Exact Snowflake Snowflake Minus / X-divertor
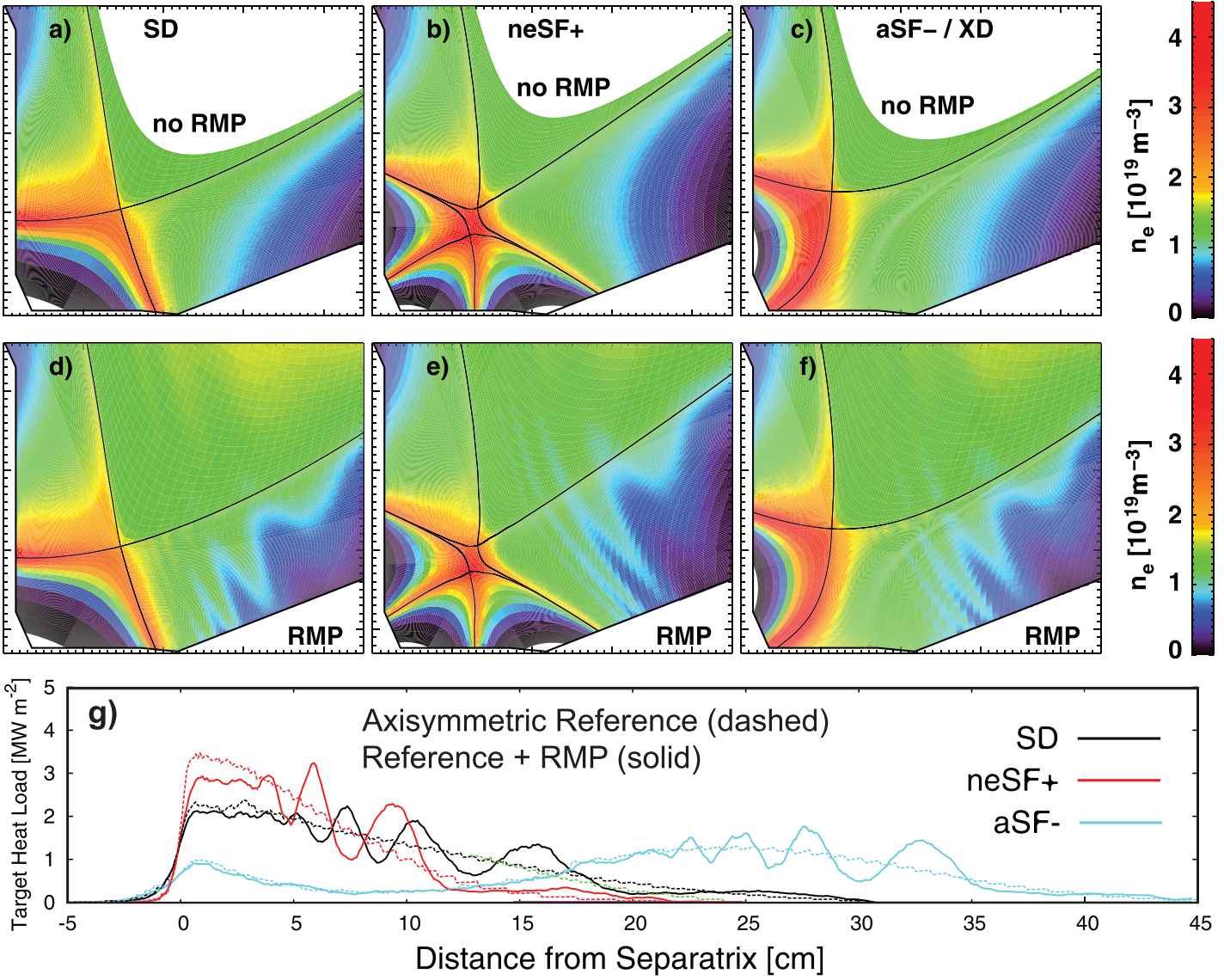

Figure 19. Comparison of EMC3-EIRENE simulations of the standard, near-exact snowflake, and X divertors for NSTX-U. Panels $(a)-(c)$ show the equilibria, panels $(d)-(f)$ show the impact of applied magnetic perturbations, and panel $(g)$ shows heat flux profiles for the configurations with and without RMP applied (reprinted from [45], with the permission of AIP Publishing).

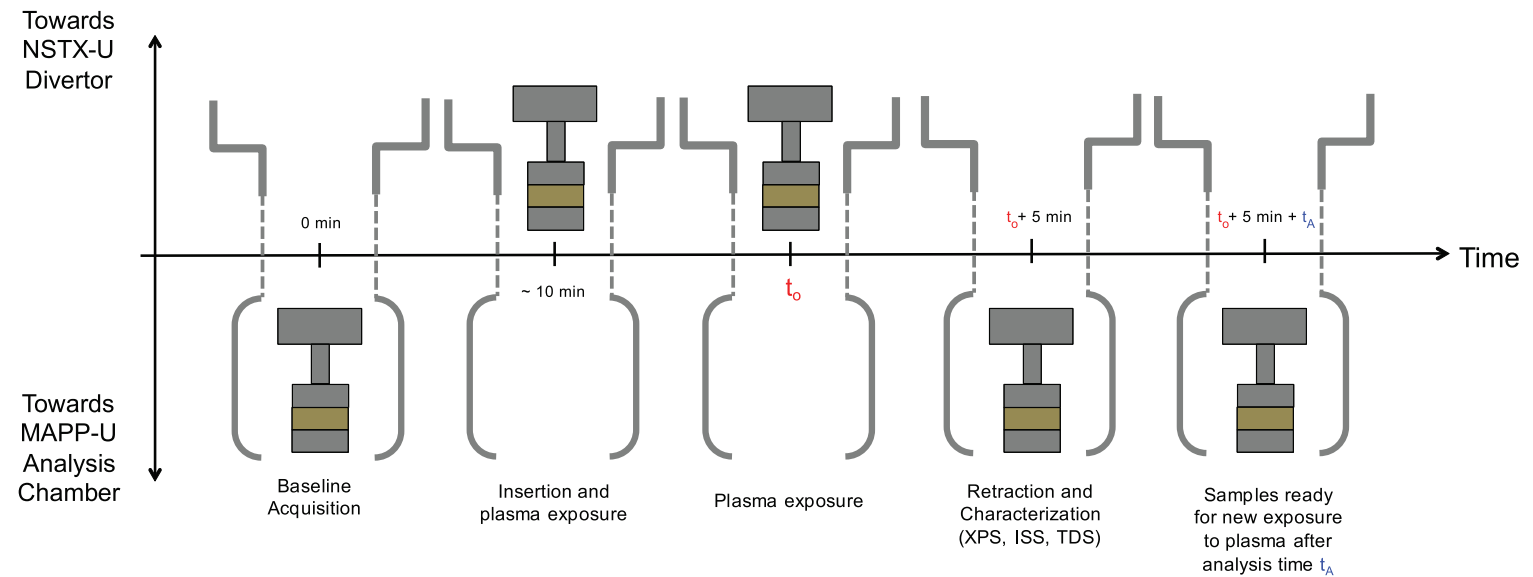

Figure 20. Sequence of between-shot operation of MAPP in NSTX-U.

ATJ graphite samples, one titanium-zirconium-molybdenum (TZM) alloy sample, and one Au sample-the latter used for energy calibration. One of the ATJ samples remained on the MAPP probe head through the entire campaign to retain the overall net surface chemistry history and enable comparisons for post-run studies. The remaining samples were replaced several times during the campaign for offline laboratory studies and evaluation of reproducibility of sample exposures throughout the run. The MAPP operational sequence is shown graphically in figure 20.

An important application of MAPP for the first run campaign of NSTX-U was to characterize the boron deposition from a new deuterated tri-methyl boron (dTMB) boronization system [48]. When new samples were installed, baseline preplasma exposure XPS data were collected, and the samples were then exposed to boronization in NSTX-U followed by 


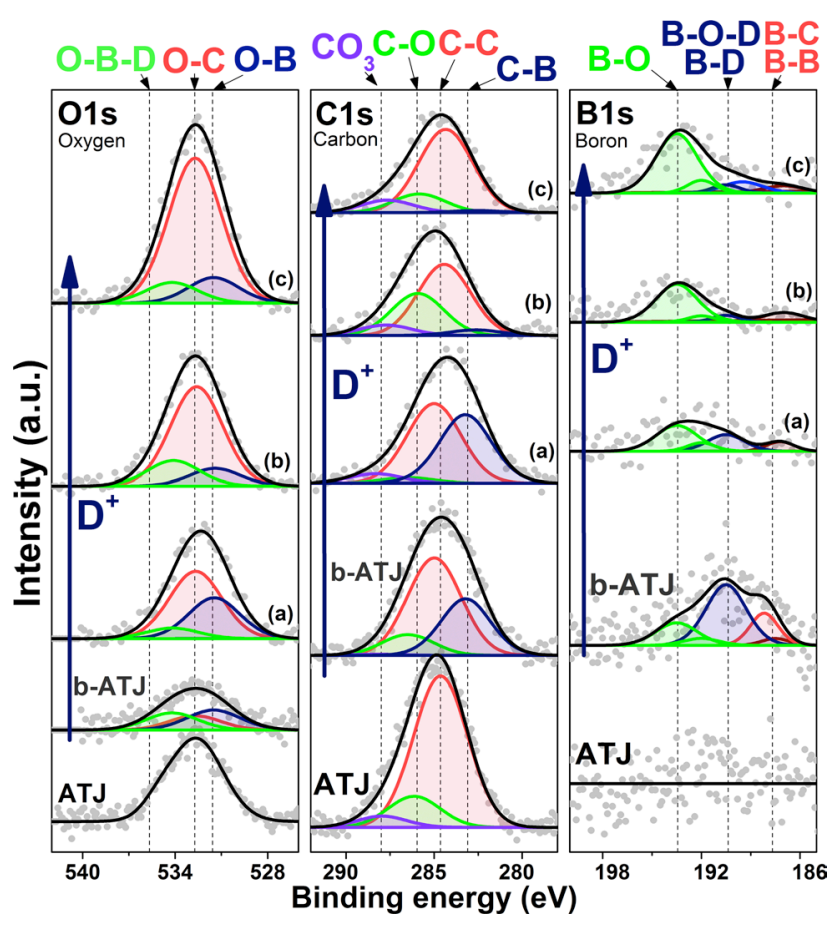

Figure 21. XPS peaks deconvolution for three region scans of boronized graphite exposed to NSTX-U plasmas over $(a) 3,(b) 8$, and $(c) 12$ days. The traces with the label 'ATJ' are the ATJ graphite baselines.

additional XPS measurements with MAPP. The samples were then inserted for one day plasma exposures. A set of XPS data was collected at the end of each day of plasma operations. This methodology of data collection improved the time resolution by two orders of magnitude compared with earlier plasma facing component (PFC) characterization methodologies which consisted of post mortem analysis of witness samples retrieved after months of an experimental campaign. Figure 21 shows a summary example of the evolution of the surfaces of one of the ATJ samples from its baseline measurement through several days of plasma exposure. The untreated ATJ sample, labeled 'ATJ' in figure 21, shows a dominant $\mathrm{C} 1 s$ peak where the $\mathrm{C}-\mathrm{C}$ interactions are the predominant state. The sample at this point was almost $90 \%$ carbon, the remaining percentage being oxygen. After boronization ('b-ATJ' in figure 21), the oxygen concentration dropped below 5\%, as did the carbon, decreasing to $57 \%$. The boron concentration was over $30 \%$ for all the boronizations. With exposure to the plasma, the oxygen concentration increased as the days progressed as shown by figure 21 panel O1s. This oxidation can also be seen in figure 21 panel $\mathrm{B} 1 s$, where the $\mathrm{B}-\mathrm{O}$ component increased in area while the $\mathrm{B}-\mathrm{B}$ and $\mathrm{B}-\mathrm{C}$ components decreased. Additionally, the $\mathrm{B}-\mathrm{C}$ interaction in the $\mathrm{C} 1 s$ panel decreased, which could be evidence of sputtering and material migration. In the final day before a new boronization, i.e. corresponding to the traces labeled ' $(c)$ ' in figure 21 , the oxygen total peak is the largest, while almost all the area of the $\mathrm{B} 1 s$ envelope belongs to the $\mathrm{B}-\mathrm{O}$ bonds. That day, the oxygen concentration was close to $20 \%$, while the boron dropped to $21 \%$ and the carbon was 59\%. A similar behavior was observed for all of the boronizations in which a full dTMB bottle was used.

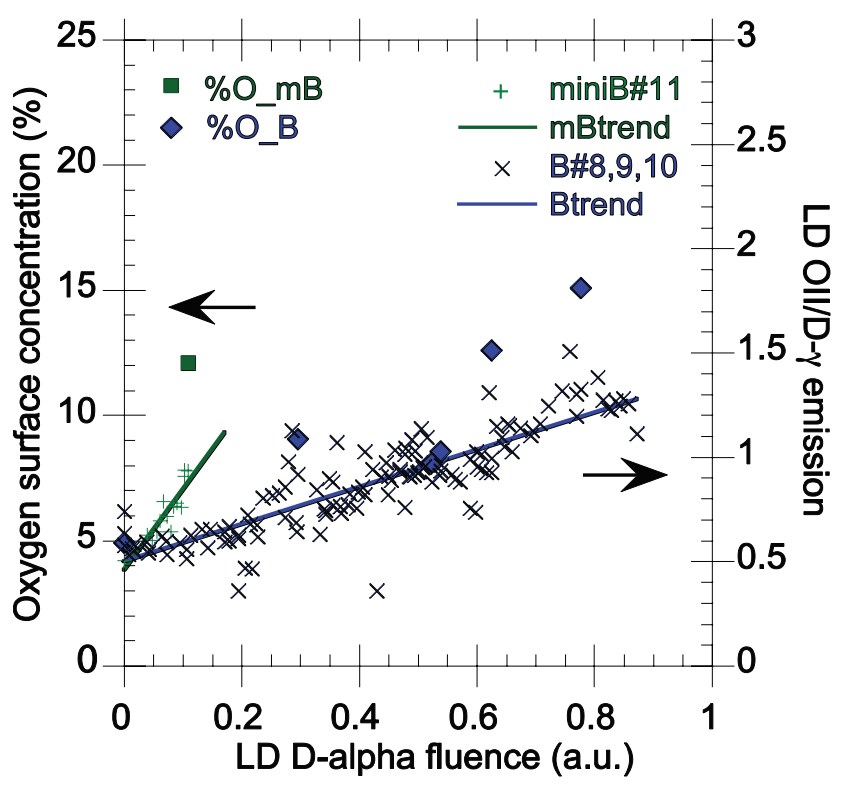

Figure 22. Fast rise in OII emission after mini-boronization using 1.5 dTMB (shown as + , miniB \#11), compared to slower rise after 'full-bottle' boronizations (shown as $x, \mathrm{~B} \# 8,9,10$ ). The fast and slow $\mathrm{O}$ II emission rise is consistent with the respective rise in surface atomic oxygen concentration as measured by MAPP XPS shown as and $\square$, respectively, all plotted against the cumulative lower divertor $D-\alpha$ fluence. The $\mathrm{mBtrend}$ and Btrend lines are linear fits to O II emission data. (Reprinted from [48], Copyright 2016, with permission from Elsevier.)

After initial improvement in wall conditions, higher power discharges started challenging PFCs and the frequency of full bottle boronizations was increased to support $\mathrm{H}$-mode discharge development. 'Mini-boronizations' (1/4-1/5th of a dTMB bottle every night) were also attempted to assess the possibility of achieving more constant daily wall conditions. The surface chemistry was tracked over several run weeks and months by MAPP. XPS measurements showed that the surface oxygen concentration measured the day after a full boronization was $4 \%-9 \%$ and rose up to $26 \%$ after $142 \mathrm{~s}$ of cumulative plasma exposure. During a 2 week maintenance break, the surface $\mathrm{O}$ concentration rose from $4 \%$ after boronization \#6, to $11 \%$ following venting the vessel to argon and several He-GDC tile conditioning procedures.

As shown in figure 22, the effect of the changing surface conditions on plasma impurities was monitored by filterscopes that viewed the lower divertor and observed O II $441 \mathrm{~nm}, D-\gamma$ and $D$ - $\alpha$ emission lines. The O II emission normalized to $D$ $\gamma$ was typically lower by a factor of 5 after boronization but subsequently rose back to its original value. Similar behavior was observed in the surface oxygen concentration as measured by MAPP XPS O $1 s$ line. The fast and slow O II emission rise after mini (1.5 g-TMB) and full bottle (9 g-TMB) boronizations were correlated with the fast and slow respectively rise in plasma facing surface atomic oxygen concentration as measured by MAPP XPS (see figure 21). While this behavior is not unexpected, the result is of interest as it is one of the first direct correlations of plasma parameters with measurements of the plasma facing surface composition. Mini-boronizations resulted in oxygen levels dropping to the same level as after 
(a)
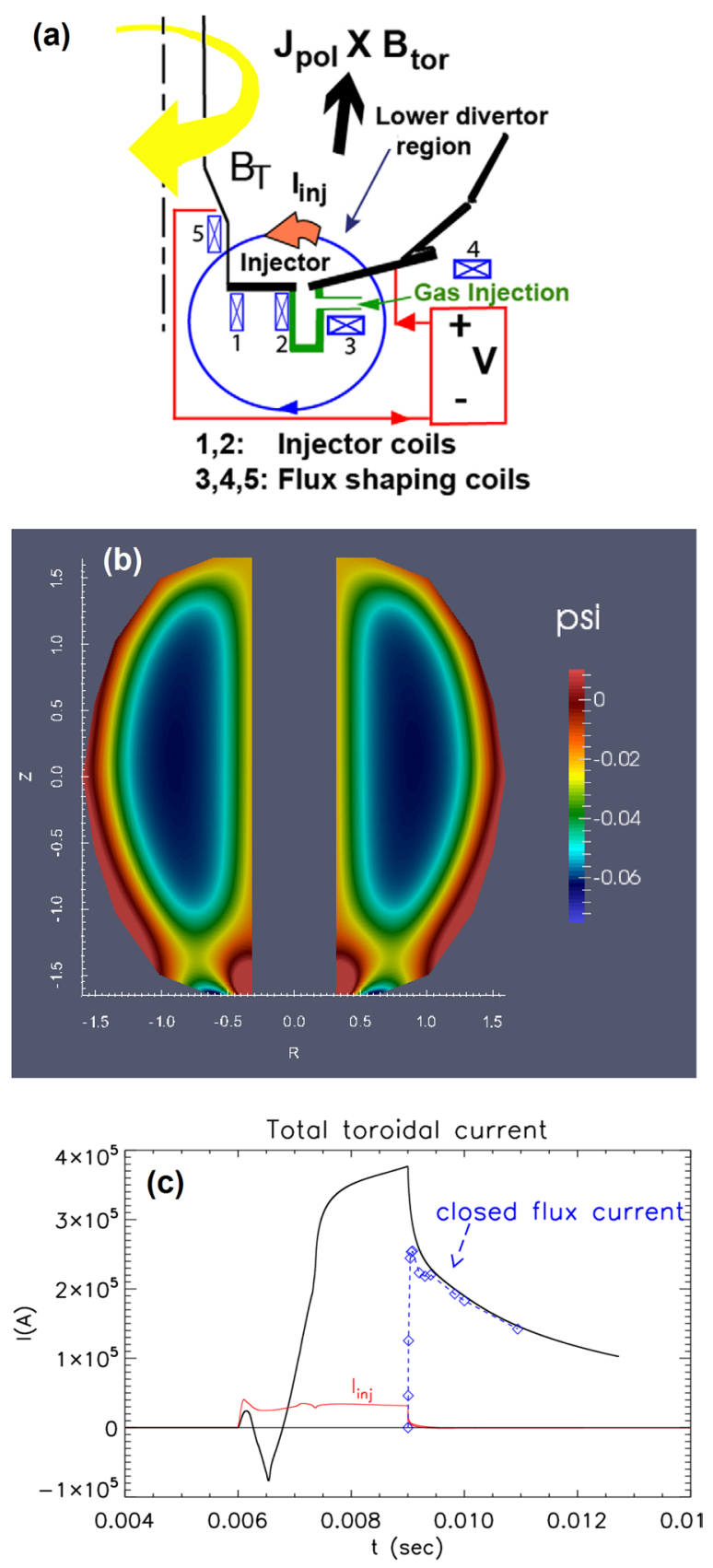

Figure 23. (a) Drawing showing the main components in NSTX-U required for plasma start-up using CHI. (b) NIMROD-simulated CHI poloidal flux, and (c) plasma current, injector current (red), and closed flux currents (blue) (Reproduced courtesy of IAEA. Figure from [51]. Copyright 2016 IAEA.).

full boronizations but exhibited a more rapid increase back to high oxygen levels. Operationally, mini-boronizations helped maintain oxygen levels in a range that made plasmas more reproducible from day to day, but the highest-performance $\mathrm{H}$-modes were obtained after full boronization.

\subsection{Solenoid-free plasma start-up}

3.5.1. Coaxial helicity injection simulations. Future fusion applications of the ST such as an FNSF [4, 49, 50] may require plasma initiation and sustainment without a central solenoid. Coaxial helicity injection (CHI) is a leading candidate method of non-solenoidal plasma start-up. Device commissioning and scenario development were highest priority during the first run campaign of NSTX-U and CHI experiments were not performed during initial operation. However, extensive CHI modelling for NSTX-U has been performed, and recent NIMROD simulations have obtained very high levels of closed flux for CHI initiation in NSTX-U geometry [51] due to improved $\mathrm{PF}$ coil placement, higher PF current, and improved ability to generate narrow flux footprints in the lower divertor.

An example of a simulation which obtained large flux closure is shown in figure 23. In this simulation, currents are driven in the injector flux shaping coils to bring the injector flux footprints close together. Figure 23(a) shows the main components in NSTX-U required for plasma start-up using CHI. The initial PF, the injector-flux (shown by the blue ellipse), connecting the inner and outer divertor plates in the injector region is produced using the lower divertor coils (shown with numbers 1,2). The primary injector coil (PF1CL) and the flux shaping coils (PF2L and PF1AL) used in the simulations are shown with numbers 2,3 and 5, respectively. To obtain a narrow injector flux footprint, the currents in the flux shaping coils are in the opposite direction of current in the primary injector flux coil (which has current in the same direction as $I_{P}$ ). As the injector flux footprint (see figure 23(b)) is made narrower, force balance requires a larger injector current since the field line tension increases. This is evident in figure 23(c) where a relatively high injector current of $34 \mathrm{kA}$ is needed for a narrow flux footprint versus $16 \mathrm{kA}$ injector current needed for a wide-footprint (not shown). This narrow footprint scenario generates $\sim 240 \mathrm{kA}$ of closed-flux current and using the full kA. turn capability of the injector coil is projected to generate $\sim 400 \mathrm{kA}$ of closed flux current. These simulations also show that the closed flux generation following injector voltage and current reduction can occur both during plasmoid mediated reconnection $[52,53]$ or a simpler SweetParker type reconnection [51]. This plasmoid-mediated reconnection accelerates the reconnection process which increases the closed-flux current fraction and increases the likelihood that sufficient plasma start-up current can be generated to be coupled to auxiliary heating and current-drive for plasma current ramp-up [54].

\section{Summary}

The NSTX facility has undergone a major upgrade and the new NSTX Upgrade (NSTX-U) was completed in the summer of 2015. First plasma was subsequently achieved, diagnostic and control systems commissioned, the H-mode accessed, magnetic EFs identified and mitigated, and the first physics research campaign carried out. NSTX-U surpassed NSTX-record pulse-durations and toroidal fields, and high-performance 1 MA H-mode plasmas comparable to the best of NSTX have been sustained near and slightly above the $n=1$ no-wall stability limit and with $\mathrm{H}_{98 y, 2} \geqslant 1$. Transport and turbulence studies in L-mode and $\mathrm{H}$-mode plasmas were initiated and several types of micro-instabilities were identified with both the BES diagnostic and in GYRO simulations. The new second more tangential NBI was observed to significantly 
modify the stability of two types of Alfven eigenmodes and the first empirical evidence that the second NBI can provide offaxis deposition was obtained. Substantial progress was made in offline disruption forecasting via improved identification of rotating MHD modes, characterization of RWM disruptions, and in the development of a reduced kinetic model for RWM stability. Lastly, the MAPP was utilized on NSTX-U for the first time enabling detailed assessments of the correlation between boronized wall conditions and plasma performance in NSTX-U.

\section{Acknowledgments}

The NSTX-U engineering, operations, and research teams are acknowledged for their relentless and excellent work that enabled the results described in this paper. This work was supported primarily by the US DOE under Contract Number DE-AC02-09CH11466. The digital data for this paper can be found in: http://arks.princeton.edu/ark:/88435/ dsp016w924f316.

\section{References}

[1] Peng M. et al 2005 Plasma Phys. Control. Fusion 47 B263-83

[2] Peng M. et al 2009 Fusion Sci. Technol. 56957

[3] Menard J.E. et al 2011 Nucl. Fusion 51103014

[4] Menard J.E. et al 2016 Nucl. Fusion 56106023

[5] Menard J.E. et al 2012 Nucl. Fusion 52083015

[6] Gerhardt S.P. et al 2012 Nucl. Fusion 52083020

[7] Ono M. et al 2015 Nucl. Fusion 55073007

[8] Menard J.E. et al 2010 Nucl. Fusion 50045008

[9] Menard J.E. et al 2005 Nucl. Fusion 45539

[10] Menard J.E. et al 2004 Phys. Plasmas 11639

[11] Berkery J.W. et al 2015 Nucl. Fusion 55123007

[12] Heidbrink W. et al 2008 Phys. Plasmas 15055501

[13] Fredrickson E. 2016 Parametric dependence of EPMs in NSTX Preprint: 2016 IAEA Fusion Energy Conf. (Kyoto, Japan, 17-22 October 2016) Poster EX/P4-41 (https:// nucleus.iaea.org/sites/fusionportal/Shared\%20Documents/ FEC\%202016/fec2016-preprints/preprint0057.pdf)

[14] Belova E. 2016 Coupling of neutral-beam-driven compressional Alfvén Eigenmodes to kinetic Alfvén waves in NSTX and energy channelling Preprint: 2016 IAEA Fusion Energy Conf. (Kyoto, Japan, 17-22 October 2016) (https://nucleus. iaea.org/sites/fusionportal/Shared\%20Documents/FEC $\% 20$ 2016/fec2016-preprints/preprint0576.pdf)

[15] Appert K. et al 1982 Plasma Phys. 241147

[16] Gorelenkov N.N. et al 2003 Nucl. Fusion 43228

[17] Goldston R.J. et al 1981 J. Comput. Phys. 4361

[18] Fredrickson E.D. et al 2016 Suppression of Alfvén modes through additional beam heating Phys. Rev. Lett. submitted

[19] Stutman D. et al 2009 Phys. Rev. Lett. 102115002

[20] Wong K.-L. et al 1991 Phys. Rev. Lett. 661874

[21] Heidbrink W. et al 1991 Nucl. Fusion 311635

[22] Wong H.V. et al 1999 Phys. Lett. A 251126

[23] Boyer M.D. et al 2015 Nucl. Fusion 55053033

[24] Goumiri I.R. et al 2016 Nucl. Fusion 56036023

[25] Podesta M. and Bell R.E. 2016 Plasma Phys. Control. Fusion 58125016

[26] Kaye S.M. et al 2006 Nucl. Fusion 46848

[27] Kaye S.M. et al 1997 Nucl. Fusion 371303

[28] Kaye S.M. et al 2007 Nucl. Fusion 47499

[29] Rebut P.H. and Brusati M. 1986 Plasma Phys. Control. Fusion 28113
[30] Rebut P.H. et al 1988 Proc. 12th IAEA Conf. on Plasma Physics and Controlled Nuclear Fusion Research (Nice) Paper IAEA-CN-50/D-4-1 (http://iaea.org/inis/ collection/NCLCollectionStore/_Public/21/008/21008733. pdf \#page $=209$ )

[31] Kaye S.M. et al 2014 Phys. Plasmas 21082510

[32] Smith D.R. et al 2012 Rev. Sci. Instrum. 83 10D502

[33] Ren Y. 2016 Exploring the regime of validity of global gyrokinetic simulations with spherical tokamak plasmas Preprint: 2016 IAEA Fusion Energy Conf. (Kyoto, Japan, 17-22 October 2016) Poster EX/P4-35 (https://nucleus. iaea.org/sites/fusionportal/Shared\%20Documents/FEC\%20 2016/fec2016-preprints/preprint0735.pdf)

[34] Candy J. and Waltz R.E. 2003 J. Comput. Phys. 186545

[35] Diallo A. 2016 Energy exchange dynamics across L-H transitions in NSTX Preprint: 2016 IAEA Fusion Energy Conf. (Kyoto, Japan, 17-22 October 2016) oral presentation EX/5-3 (https://nucleus.iaea.org/sites/fusionportal/ Shared\%20Documents/FEC\%202016/fec2016-preprints/ preprint0626.pdf)

[36] Stoltzfus-Dueck T. 2016 Phys. Plasmas 23054505

[37] Sontag A.C. et al 2005 Phys. Plasmas 12056112

[38] Sabbagh S.A. 2006 Phys. Rev. Lett. 97045004

[39] Berkery J. 2016 Characterization and forecasting of unstable resistive wall modes in NSTX and NSTX-U Preprint: 2016 IAEA Fusion Energy Conf. (Kyoto, Japan, 17-22 October 2016) Poster EX/P4-34 (https://nucleus.iaea.org/sites/ fusionportal/Shared\%20Documents/FEC\%202016/fec2016preprints/preprint0281.pdf)

[40] Soukhanovskii V. 2016 Divertor configuration effects on pedestal stability and edge localized modes in NSTX and DIII-D Preprint: 2016 IAEA Fusion Energy Conf. (Kyoto, Japan, 17-22 October 2016) poster EX/P3-30 (https:// nucleus.iaea.org/sites/fusionportal/Shared\%20Documents/ FEC\%202016/fec2016-preprints/preprint0376.pdf)

[41] Lore J. 2016 Pedestal-to-wall 3D fluid transport simulations on DIII-D and NSTX Preprint: 2016 IAEA Fusion Energy Conf. (Kyoto, Japan, 17-22 October 2016) Poster TH/P6-12 (https:// nucleus.iaea.org/sites/fusionportal/Shared\%20Documents/ FEC\%202016/fec2016-preprints/preprint0378.pdf)

[42] Ahn J.-W. 2016 Shielding and amplification of nonaxisymmetric divertor heat flux by plasma response to applied 3D fields in NSTX and KSTAR Int. Atomic Energy Agency Fusion Energy Conf. (Kyoto, Japan, 17-22 October 2016) Poster EX/P4-30 (https://nucleus.iaea.org/sites/ fusionportal/Shared\%20Documents/FEC\%202016/fec2016preprints/preprint0628.pdf)

[43] Ryutov D. et al 2010 Plasma Phys. Control. Fusion 52105001

[44] Kotschenreuther M. et al 2013 Phys. Plasmas 20102507

[45] Frerichs H. et al 2016 Phys. Plasmas 23062517

[46] Heim B. et al 2012 IEEE Trans. Plasma Sci. 40735

[47] Bedoya F. et al 2016 Initial studies of plasma facing component surface conditioning in the national spherical Tokamak experiment upgrade with the materials analysis particle probe Nucl. Mater. Energy submitted

[48] Skinner C.H. et al 2017 Advances in boronization on NSTX-upgrade Nucl. Mater. Energy (https://doi.org/10.1016/j.nme.2016.11.024)

[49] Raman R. et al 2015 Fusion Sci. Technol. 68674

[50] Raman R. et al 2016 J. Fusion Energy 35 34-40

[51] Ebrahimi F. et al 2016 Nucl. Fusion 56044002

[52] Ebrahimi F. et al 2015 Phys. Rev. Lett. 4205003

[53] Ebrahimi F. 2016 Physics of flux closure during plasmoidmediated reconnection in coaxial helicity injection Preprint: 2016 IAEA Fusion Energy Conf. (Kyoto, Japan, 17-22 October 2016) poster TH/P1-2 (https://nucleus.iaea. org/sites/fusionportal/Shared\%20Documents/FEC\%20 2016/fec2016-preprints/preprint0395.pdf)

[54] Poli F. et al 2015 Nucl. Fusion 55123011 